\section{Chronic Kidney Disease}

1

\section{Sugar and Artificially Sweetened Soda and Chronic Kidney Disease: A Meta-Analysis}

W. Cheungpasitporn ', C. Thongprayoon ${ }^{1}$, O.A. O'Corragain ${ }^{2}$, P.J. Edmonds ${ }^{3}$, W. Kittanamongkolchai ${ }^{1}$, S.B. Erickson ${ }^{1}$

${ }^{1}$ Nephrology and Hypertension, Mayo Clinic, Rochester, MN, USA; ${ }^{2}$ Medicine, University College Cork, Cork, Ireland;

${ }^{3}$ Medicine, SUNY Upstate Medical University, Syracuse, NY, USA

Background: The risk of chronic kidney disease (CKD) in patients who regularly drink soda is controversial. The objective of this meta-analysis was to evaluate the associations between consumption of sugar and artificially sweetened soda and CKD.

Methods: A literature search was performed using MEDLINE, EMBASE, and Cochrane Database of Systematic Reviews from inception until June 2014. Studies that reported odd ratios or hazard ratios comparing the risk of $\mathrm{CKD}$ in patients consuming significant amount of either sugar or artificially sweetened soda versus those who did not consume soda were included. Pooled risk ratios (RR) and 95\% confidence interval (CI) were calculated using a random-effect, generic inverse variance method.

Results: Five studies were included in our analysis to assess the association between consumption of sugar sweetened soda and $\mathrm{CKD}$. The pooled RR of CKD in patients consuming sugar-sweetened soda was 1.58 (95\% CI, 1.00-2.49). Four studies were selected to assess the association between consumption of artificially sweetened soda and CKD. The pooled RR of CKD in patients consuming artificially sweetened soda was 1.33 (95\% CI, $0.82-2.15)$.

Conclusions: Our study demonstrates statistically significant increased risks of CKD in patients consuming sugar sweetened soda, but not in patients consuming artificially sweetened soda. This finding suggests that sugar sweetened soda consumption is associated with CKD and may impact clinical management and primary prevention of CKD in high-risk patients.

Keywords: Chronic kidney disease, Soft drink, Soda.

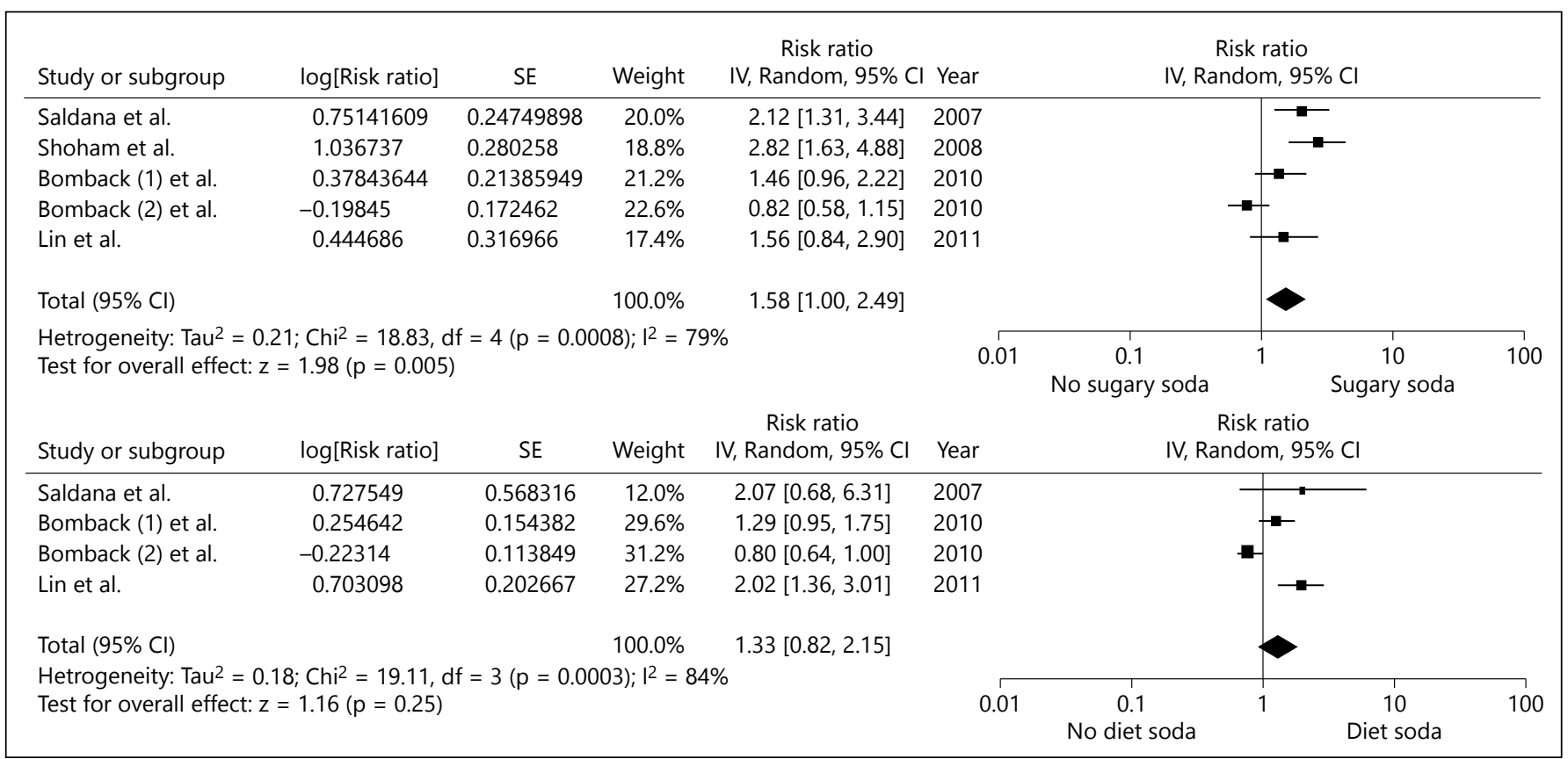

Fig. 1. (for Abstract 1).

\begin{tabular}{|c|c|}
\hline KARGER 125 & $\begin{array}{l}\text { (c) } 2015 \text { S. Karger AG, Basel } \\
0253-5068 / 15 / 0393-0151 \$ 39.50 / 0\end{array}$ \\
\hline $\begin{array}{l}\text { Fax +4161306 } 1234 \\
\text { E-Mail karger@karger.ch } \\
\text { www.karger.com }\end{array}$ & $\begin{array}{l}\text { Accessible online at: } \\
\text { www.karger.com/bpu }\end{array}$ \\
\hline
\end{tabular}


2

\section{Is High Alcohol Consumption a Risk Factor for End Stage Renal Disease? A Meta-Analysis}

B.A. Brabec ${ }^{1}$, W. Cheungpasitporn' ${ }^{1}$, C. Thongprayoon', W. Kittanamongkolchai ${ }^{1}$, P.J. Edmonds' ${ }^{2}$, S.B. Erickson ${ }^{1}$

${ }^{1}$ Nephrology and Hypertension, Mayo Clinic, Rochester, MN, USA; ${ }^{2}$ Medicine, SUNY Upstate Medical University, Syracuse, NY, USA

Background: The risk of end stage renal disease (ESRD) in patients with high alcohol consumption is unclear. The objective of this meta-analysis was to evaluate the associations between high alcohol consumption and ESRD.

Methods: A literature search was performed using MEDLINE, EMBASE, and Cochrane Database of Systematic Reviews from inception through August, 2014. Studies that reported odd ratios, relative risks or hazard ratios comparing the risk of ESRD in patients consuming high amount of alcohol versus those who did not consume alcohol were included. Pooled risk ratios (RR) and 95\% confidence interval (CI) were calculated using a randomeffect, generic inverse variance method.

Results: Five studies ( 3 cohort and 2 case-control studies) with 80,583 patients were included in our analysis to assess the association between high alcohol consumption and ESRD. The pooled RR of ESRD in patients with high alcohol consumption was 1.00 (95\% CI, 0.55-1.82) as shown in figure. The pooled RRs for developing ESRD related to hypertension (HTN), diabetes mellitus (DM) and HTN or DN were 2.23 (95\% CI, 0.90-5.52), 0.32 (95\% CI, 0.05$2.27)$ and $1.07(0.26-4.31)$, respectively. The post hoc analysis assessing the sex-specific association between high alcohol consumption and ESRD demonstrated pooled RR of 1.43 (95\% CI, $0.24-8.55)$ in males and 2.1 (95\% CI, 0.1-103.7) in females.

Conclusions: Our study demonstrates no significant association between high alcohol consumption and the risk for developing ESRD, both in males and females. High alcohol consumption is not significantly associated with both HTN and DM related ESRD.

Keywords: End stage renal disease, High alcohol consumption, Meta-analysis.
3

\section{The Association between Proteinuria and High Alcohol Consumption: A Meta-Analysis}

\author{
W. Kittanamongkolchai, W. Cheungpasitporn, C. Thongprayoon, \\ S.B. Erickson
}

Nephrology and Hypertension, Mayo Clinic, Rochester, MN, USA

Background: The risk of proteinuria in patients with high alcohol consumption is controversial. The objective of this meta-analysis was to evaluate the association between high alcohol consumption and proteinuria.

Methods: A literature search was performed using MEDLINE, EMBASE, and the Cochrane Database of Systematic Reviews from inception through August 2014. Studies that reported odd ratios, relative risks, or hazard ratios comparing the risk of CKD in patients consuming high amount of alcohol versus those who did not consume alcohol were included. Pooled risk ratios (RR) and $95 \%$ confidence intervals (CI) were calculated using a randomeffect, generic inverse variance method.

Results: Four study samples (all cohort studies) with 140,686 patients were included in our analysis to assess the association between high alcohol consumption and proteinuria. The pooled $\mathrm{RR}$ of proteinuria in patients with high alcohol consumption was 0.85 (95\% CI, 0.62-1.17).

Conclusions: Our study demonstrates no significant association between high alcohol consumption and risk for developing proteinuria.

Keywords: Proteinuria, Alcohol consumption, Meta-analysis.

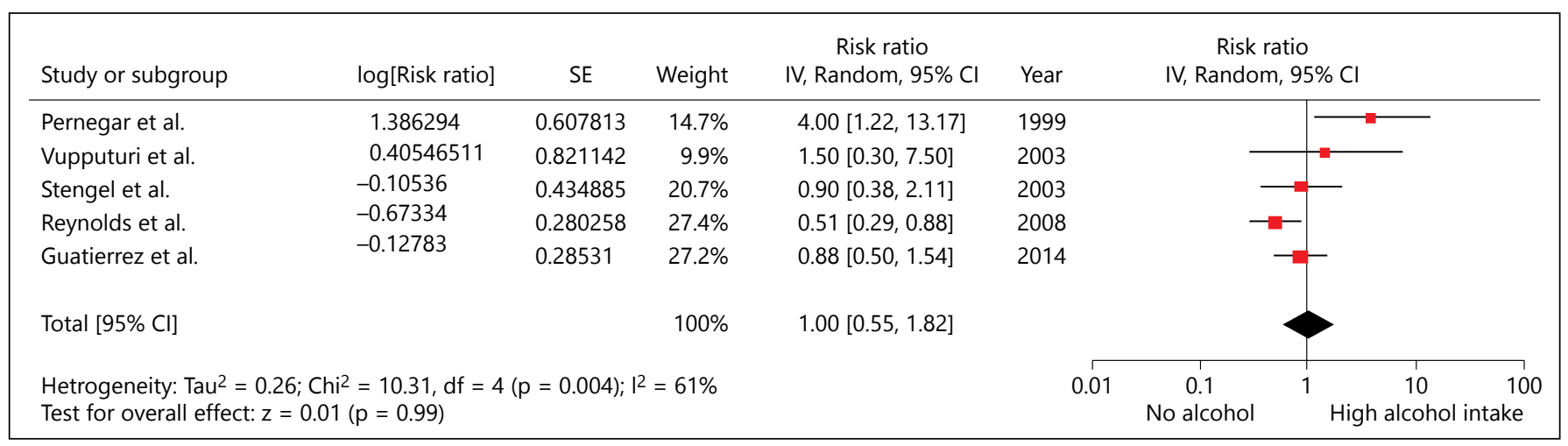

Fig. 1. (for Abstract 2). 


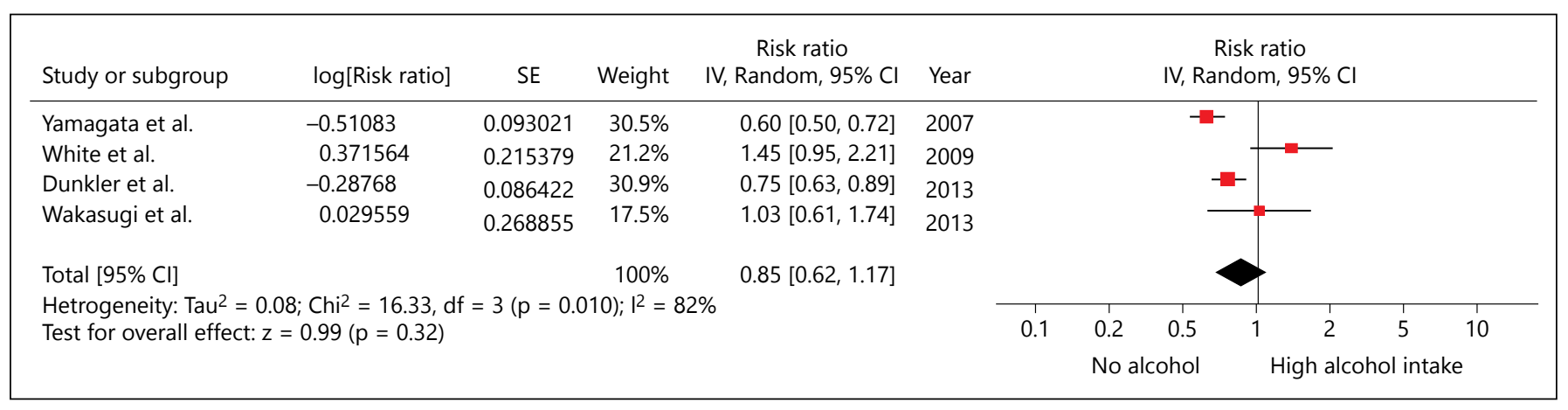

Fig. 1. (for Abstract 3).

\section{4 \\ Effects of Statins on Renal Outcome in Chronic Kidney Disease Patients: A Systemic Review and Meta-Analysis

\author{
A. Sanguankeo', S. Upala', W. Cheungpasitporn ${ }^{2}$, P. Ungprasert ${ }^{2}$, \\ E.L. Knight ${ }^{1}$ \\ ${ }^{1}$ Internal Medicine, Bassett Medical Center and Columbia \\ University College of Physicians and Surgeons, Cooperstown, \\ NY, USA; ${ }^{2}$ Medicine, Mayo Clinic, Rochester, MN, USA
}

Background: HMG CoA reductase inhibitors (statins) are known to prevent cardiovascular disease and improve lipid profiles. However, the effects of statins on renal outcomes including decline in estimated glomerular filtration rate (eGFR) and proteinuria in patients with chronic kidney disease (CKD) are controversial. The objective of this meta-analysis was to evaluate the impact of statins on renal outcomes in patients with CKD.

Methods: We comprehensively searched the databases of PubMed/MEDLINE, EMBASE, and CENTRAL. The inclusion criteria were published articles in patients with an eGFR less than $60 \mathrm{ml} / \mathrm{min} / 1.73 \mathrm{~m}^{2}$ whom received statins for at least six months and compared statin therapy to a placebo or control. The primary outcome was the difference in the change of eGFR and proteinuria between the two groups. Two authors independently assessed the quality of the articles and extracted the data. A quantitative data analysis comparing the two groups was performed using the Review Manager 5.3 software, and a subgroup analysis examining statin intensity was performed.

Results: From 142 full-text articles, 9 studies with a total of 7,581 participants were included in the meta-analysis. Overall, there was no significant difference in total change of eGFR (mean difference $(\mathrm{MD})=0.34 \mathrm{ml} / \mathrm{min} / 1.73 \mathrm{~m}^{2}, 95 \% \mathrm{CI}:-0.83$ to $1.51)$ or eGFR change per year $\left(\mathrm{MD}=0.30 \mathrm{ml} / \mathrm{min} / 1.73 \mathrm{~m}^{2}, 95 \%\right.$ CI: -0.09 to 0.67$)$. In our subgroup analysis, those who received high-intensity statins, however, did have a significant improvement of eGFR with a MD of 2.99 (95\% CI: 0.25 to 5.74$) \mathrm{ml} /$ $\mathrm{min} / 1.73 \mathrm{~m}^{2}$. Compared with the control group, the statin group had a greater reduction of proteinuria with a standardized mean difference $(\mathrm{SMD})$ in change of proteinuria of -0.30 (95\% CI: -0.55 to -0.05$)$.

17th International Conference on Dialysis, Advances in CKD 2015
Conclusions: High intensity statins were found to improve total change in eGFR compared with control, but low and moderate dose statins were not found to decrease eGFR. In addition, statins were found to decrease proteinuria in patients with CKD, consistent with prior studies.

Keywords: Statins, Chronic kidney disease, Meta-analysis.

\section{5 \\ Preventing Chronic Kidney Disease and the Need for Dialysis in Most People in 10 Years}

\section{B. von Hartitzsch}

Nephrology Specialists of Oklahoma, Tulsa, OK, USA

Background: From 2001 to 2007 when I had the opportunity to correct Hemoglobin levels to normal 13-16.0 gm/dl uremic patients began to thank me for making them feel normal for the first time in years. The oxygen carrying capacity of their blood had been restored and every living cell was now functioning normally. Their uremic symptoms had disappeared.

Methods: My next task was to see if, Smoking, Obesity, Hypertension, Cardiac Disease and Diabetes all risk factors for chronic kidney disease could interfere with oxygen delivery to the tissues. Yes! all effect oxygen delivery and glucose in diabetics to the mitochondria within each cell which leads to chronic hypoxia, anaerobic respiration and failure of intracellular energy production. The result is premature aging of the cells, an increase in the daily rate of cell death and increased phagocytosis with release of IL-6, TNFa, and CRP to remove the dead cells. As the hypoxia induced phagocytosis increases in magnitude this cascade of hypoxia becomes recognized as oxidative stress and IL-6 and TNFa start to suppress erythropoiesis when creatinine clearances, Cystatin C clearances, are still above $90 \mathrm{ml} / \mathrm{min} / 1.73 \mathrm{~m}^{2}$ long before any renal injury. At this time PTH levels also start to increase as TNFa levels directly stimulate $\mathrm{PTH}$ production in parathyroid tissue.

Results: When all the risk factors anemia included are aggressively treated to normal, that means hemoglobin levels $13.5-16.5 \mathrm{gm} / \mathrm{dl}$, renal failure ceases to progress and patients with stage 3-4 chronic kidney disease remain stable for peri- 


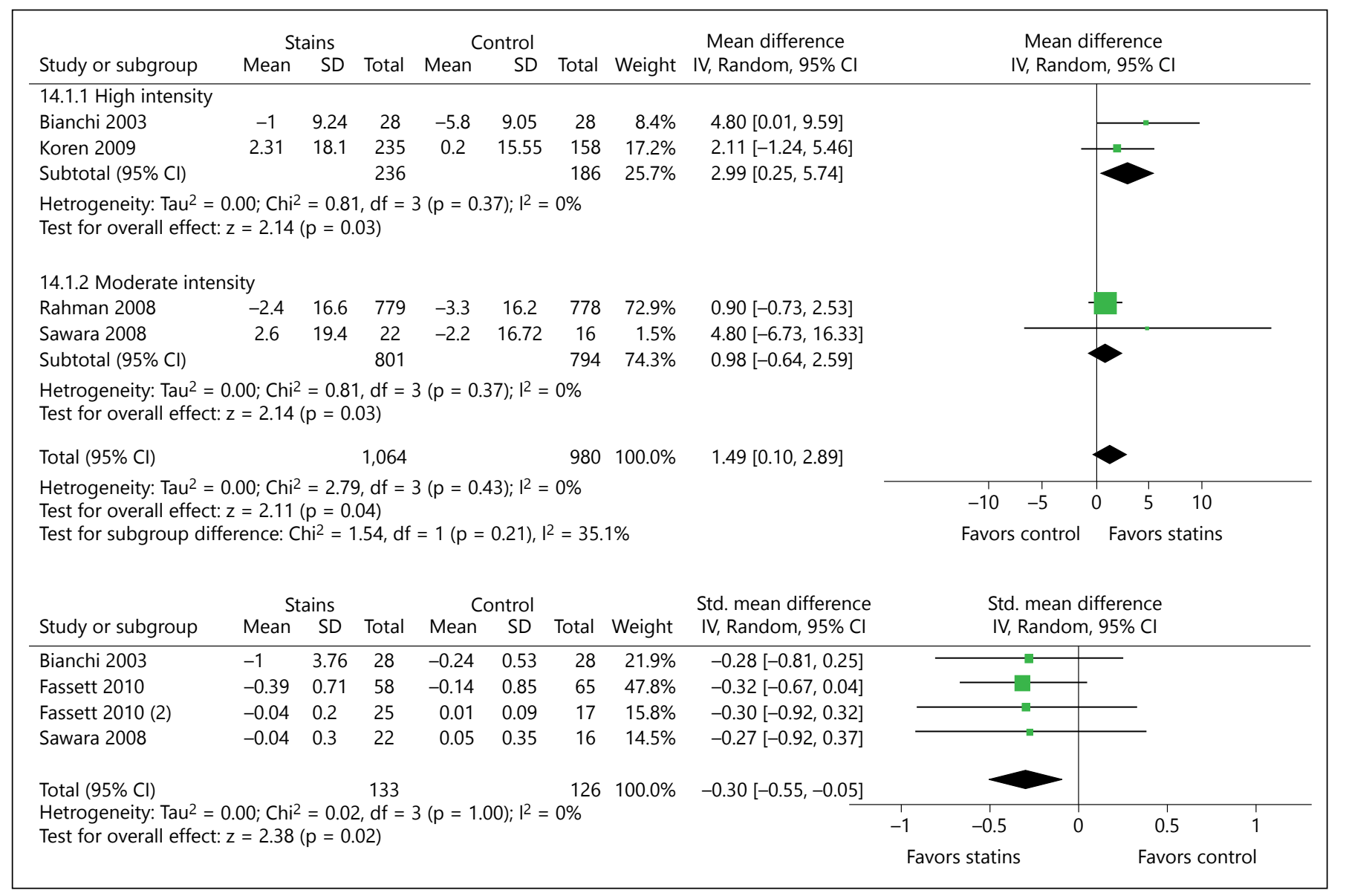

Fig. 1. (for Abstract 4).

ods of time ranging from 4.5-9.5 years average time 6.2 years. In contrast patients treated the same except for hemoglobin levels continued in the $10-12 \mathrm{gm} / \mathrm{dl}$ range progress to dialysis in less than 2 years. The ACORD Trial tells us that LVH is already present at Hemoglobin $11.9 \mathrm{gm} / \mathrm{dl}$ and ceases to progress if the anemia is corrected to at least $13.5 \mathrm{gm} / \mathrm{dl}$. Thus treatment of anemia to prevent heart disease and chronic kidney disease must start when the hemoglobin first drops out of the normal range when erythropoietin resistance will be non existent and small doses of Erythropoietin will be effective. As early as 1968 it was recognized that chronic hypoxia results in elevation of Cholesterol; it is reversed by correction of the anemia.

Conclusions: Correcting anemia with erythropoietin to correct the erythropoietin deficiency, to correct the hemoglobin levels to normal $13.5-16.0 \mathrm{gm} / \mathrm{dl}$ and to restore the oxygen carrying capacity of the blood to normal is the only way to prevent chronic kidney disease and the need for dialysis in most people in 10 years.

Keywords: Restore oxygen carrying capacity, Physiology of chronic kidney disease, Normalize hemoglobin.

\section{6 \\ Rapid Progression to End Stage Renal Disease in a Young Patient with a Sporadic ACTN4 Mutation}

A. Kakajiwala, K.E.C. Meyers

Dept. of Nephrology, The Children's Hospital of Philadelphia, Philadelphia, PA, USA

Mutations of ACTN4 cause an AD form of focal segmental glomerulosclerosis (FSGS). Presentation is usually in the teenage years or later. Onset is usually with mild proteinuria and slowly progressive renal dysfunction leading to ESRD. A five year old female presented with abdominal pain and edema. She was diagnosed with Nephrotic Syndrome complicated by spontaneous bacterial peritonitis. She had mild acute kidney injury and anemia. She did not respond to six weeks of oral glucocorticoid therapy. Renal biopsy showed a collapsing variant of FSGS and genetic studies showed a heterozygous disease-causing mutation in the ACTN4 gene (c.785C >T, p.Ser262Phe). Her parents have no ACTN4 gene mutations. No mutations were found in the NPHS2, TRPC6 and INF2 genes. She developed severe hypertension and 
posterior reversible encephalopathy syndrome (PRES) on tacrolimus and corticosteroids, which were then discontinued. She did not respond to a course of rituximab. Blood pressure was controlled with amlodipine, furosemide, losartan and metolazone. Six months after presentation she developed ESRD and started on peritoneal dialysis for hyperkalemia, acidosis and fluid overload.

ACTN4 mutations have been identified that are believed to be disease-causing. Additional polymorphisms have an unclear contribution to disease but may increase the susceptibility to and aggressiveness of FSGS. There may be additional mutations in other genes that were not identified or as yet unidentified epigenetic factors that contributed to the aggressive nature of this child's disease progression. This case illustrates a collapsing form of FSGS from a disease causing ACTN4 mutation that rapidly progressed to ESRD. A literature review failed to find a prior description of such rapid disease progression in association with an ACTN4 mutation in a child.

Keyword: ACTN4 mutation, Rapid progression of FSGS.

7

\section{CKD in Older Adults with Cardiovascular Disease}

\section{A. Champion}

Healthcare Research Insights, Lake Forest, IL, USA

Background: Patients with $\mathrm{CKD}$ are at increased risk for cardiovascular events. The objective of this analysis is to characterize the renal status of adults with cardiovascular disease compared to the general population.

Methods: Analysis of US NHANES 2011-2012 using KDIGO 2013 classification of CKD in adults in the total population and the subgroup with CVD. As CVD and CKD prevalence increase with age, this analysis focused on ages $60+$.
Results: 1,530 adults age $60+$ were eligible for analysis representing a population of 53.8 million, $23 \%$ had CVD. In the CVD group, mean age (72.5 vs. 69.6 years) and mean UACR (112.9 vs. $50.8 \mathrm{mg} / \mathrm{g}$ ) were higher and mean eGFR was lower (64.8 vs. 73.7 $\mathrm{ml} / \mathrm{min} / 1.73 \mathrm{~m}^{2}$ ) than in the total population. CVD as a percent of the total population increased from $11 \%$ in G1 to $66 \%$ in G4 and from $19 \%$ in A1 to $59 \%$ in A3. UACR $>300 \mathrm{mg} / \mathrm{g}$ (A3) was more than twice as frequent in the CVD group. In a logistic regression, after adjusting for age and UACR, CVD continued to be associated with an increased risk for reduced eGFR (G3-G5).

Conclusions: Compared to the general population age $60+$, those with CVD are older and have more renal morbidity. Individuals with CVD age $60+$ are at increased risk for reduced renal function, even after adjustment for age and UACR.

Keywords: Chronic kidney disease, Cardiovascular disease, Older adults.

8

The Need for Dialysis in Haiti: Dream or Reality?

Judith Exantus 1,2,6, Florence Desrosiers ${ }^{3}$, Alexandra Ternier², Audie Métayer ${ }^{2}$, Gérard Abel ${ }^{2,4}$, Jean-Hénold Buteau ${ }^{5}$

${ }^{1}$ Pediatrics Unit, State University Hospital, Port-au-Prince, Haïti; ${ }^{2}$ Dialysis Unit, Internal Medicine Ward, State University Hospital, Port-au-Prince, Haïti ; ${ }^{3}$ Outpatient Center Clinic, University of Illinois at Chicago, Chicago, United States of America; ${ }^{4}$ Dialysis Unit, Plurimedic, Port-au-Prince, Haïti; ${ }^{5}$ Haitian Nephrology Center, Port-au-Prince, Haïti; ${ }^{6}$ Hôpital NPFS St Damien, Tabarre, Haïti

The burden of chronic kidney diseases (CKD) is well documented nowadays according to the World Health Organization. The high prevalence of non-communicable diseases (NCD) such as hypertension, diabetes and obesity, main causes of CKD, is a

Table 1. NHANES 2011-2012 Adults Age 60+ Distribution by eGFR (CKD-EPI) and UACR categories (for Abstract 7)

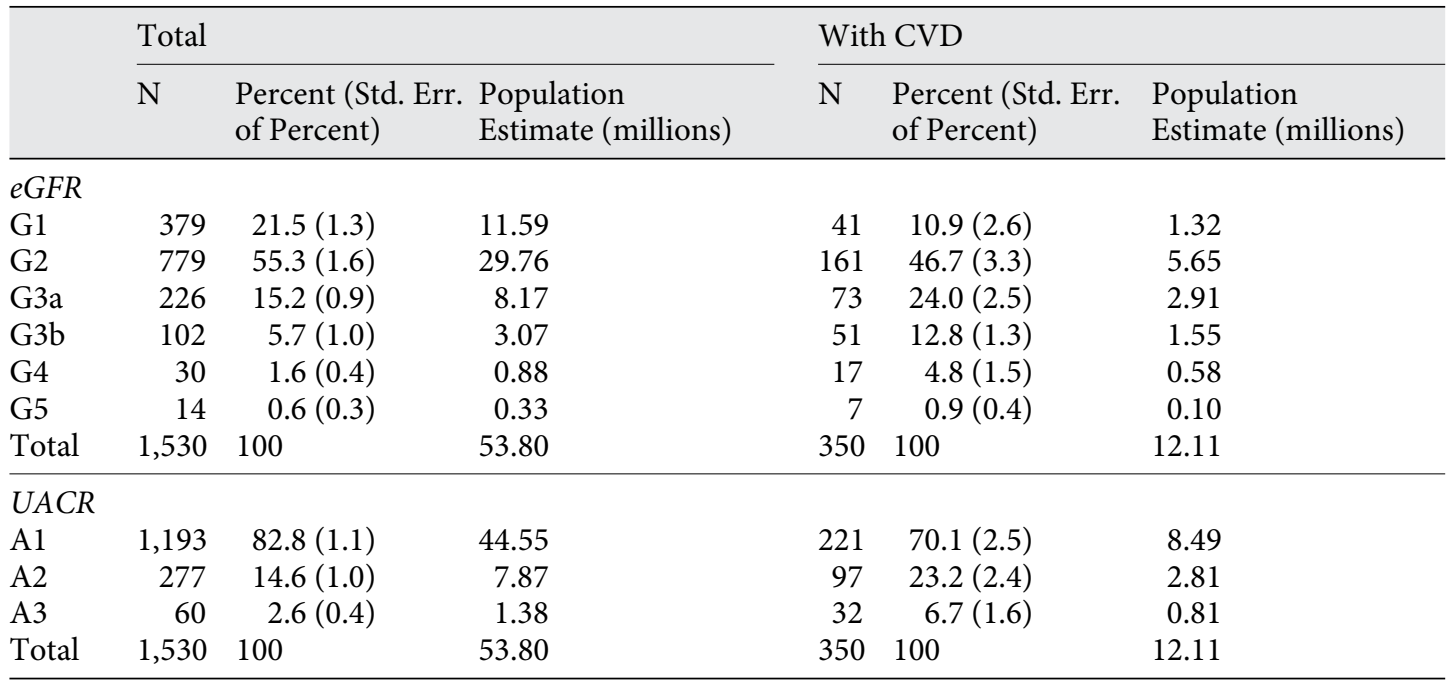


big concern of world health [1]. These NCD can progress slowly to end-stage renal disease (ESRD) and there is a massive unmet need for dialysis in low-middle income countries (LMIC) for acute and chronic renal failure.

In Haiti, chronic glomerulopathies are the leading causes of end stage renal disease (ESRD) in children. A retrospective pediatric study regarding chronic renal failure in 2010 (unpublished) showed that, for a sample of 20 children over 13 years, nephrotic syndrome, systemic lupus erythematosus and human immunodeficiency-associated nephropathy predominate (respectively $37 \%, 26 \%$ and $16 \%$ ). Only $30 \%$ (6 out of 20 ) of these children were hemodialyzed over a mean duration of 8 weeks (range $3-24)$. Considering that seventy-two (72\%) of Haitians live with less than $\$ 2$ per day [2] and few patients have access to health insurance, survival with CKD in our country can be quite stressful. Data on the weight of the dialysis and its management are scarce. There is inequity in terms of the availability and the location of dialysis units; only seventeen machines are available (all in the capital-city). Addressing the need for dialysis in Haiti is an important component in health decision-making and planning processes.

Keywords: Chronic kidney disease, Renal replacement therapy, Dialysis, Low-middle income countries, Haiti.

\section{9 \\ Comparison of Cases of Kidney Failure and End-Stage Renal Disease in the Atherosclerosis Risk in Communities Study}

C. Rebholz', J. Coresh', S. Ballew', B. McMahon', S. Whelton², E. Selvin ${ }^{1}$, M. Grams ${ }^{1}$

${ }^{1}$ Welch Center for Prevention, Epidemiology, and Clinical Research, Johns Hopkins University, Baltimore, MD, USA;

${ }^{2}$ Johns Hopkins University, Baltimore, MD, USA

Background: Linkage to the United States Renal Data System (USRDS) registry is the most common method for identifying individuals with end-stage renal disease (ESRD) in epidemiologic research, but it may underestimate the total burden of advanced kidney disease due to a lack of inclusion of untreated individuals. This study validates a kidney failure definition that includes treated and untreated disease and compares risk factors for and outcomes after kidney failure and ESRD.

Methods: In the Atherosclerosis Risk in Communities (ARIC) study, we compared risk factors for and outcomes after treated and untreated kidney failure. ESRD was defined by USRDS registry linkage. Kidney failure was defined by USRDS ESRD or kidney failure-related ICD-9/10 code from hospitalization or death. We assessed the validity of the proposed billing code algorithm for defining kidney failure to chart review.

Results: Among 11,530 participants with a median follow-up of 13 years, 508 kidney failure cases were identified, including 173 (34.1\%) from USRDS registry linkage. ESRD and kidney failure incidence, respectively, were 1.23 and 3.66 cases per 1,000 personyears in the overall population, and, among participants aged 71-75 years at baseline, were 1.35 and 6.59 cases per 1,000 personyears (figure 1). Other risk factor associations for ESRD and kidney failure were similar (sex, race, hypertension, history of

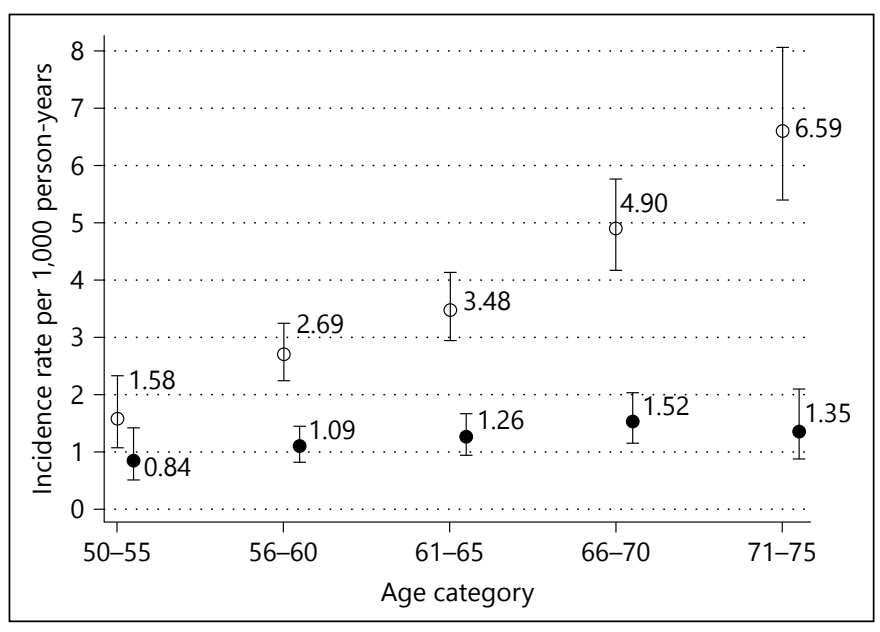

Fig. 1. Incidence rates per 1,000 person-years for kidney failure and end-stage renal disease by age categories. $\mathrm{p}<0.001$ for test of difference in hazard ratio for kidney failure vs. hazard ratio for endstage renal disease associated with age using seemingly unrelated regression. Hollow circle = kidney failure; solid circle = end-stage renal disease; bar $=95 \%$ confidence intervals. (for Abstract 9).

coronary heart disease) except for diabetes and albuminuria, which were stronger for ESRD. Survival at 1- and 5-years, respectively, were $74.0 \%$ and $24.0 \%$ for ESRD and $59.8 \%$ and $31.6 \%$ for kidney failure. In a validation of the ICD-9/10 code algorithm for defining kidney failure compared to chart review, sensitivity was $88 \%$ and specificity was $97 \%$.

Conclusions: A kidney failure definition that includes treated and untreated disease identifies substantially more cases than does USRDS registry linkage alone, particularly in older age. Future research studies might consider reporting both USRDS-identified ESRD and a more inclusive kidney failure definition.

Keywords: End-stage renal disease, Hospitalization, Validation. 


\section{Acute Kidney Injury}

10

Periprocedural Effect of Statins in the Prevention of Contrast-Induced Acute Kidney Injury: A Meta-Analysis of Randomized Controlled Trials

W. Kittanamongkolchai ${ }^{1}$, W. Cheungpasitporn', C. Thongprayoon ${ }^{1}$, P.J. Edmonds ${ }^{2}$, O.A. O'Corragain ${ }^{3}$, S.B. Erickson

${ }^{1}$ Nephrology and Hypertension, Mayo Clinic, Rochester, MN, USA; ${ }^{2}$ Medicine, SUNY Upstate Medical University, Syracuse, NY, USA; ${ }^{3}$ Medicine, University College Cork, Cork, Ireland

Background: The reports on the efficacy of statins in the prevention of contrast-induced acute kidney injury (CIAKI) remain controversial. The objective of this meta-analysis was to assess the effect of statins in the prevention of CIAKI.

Methods: Comprehensive literature searches for randomized controlled trials (RCTs) of periprocedural statin treatment for prevention of CIAKI were performed using MEDLINE, EMBASE, Cochrane Database of Systematic Reviews, Cochrane Central Register of Controlled Trials Systematic Reviews and clinicaltrials. gov from inception until May, 2014. The primary outcome was the incidence of CIAKI.

Results: Thirteen prospective RCTs were included in our analysis. Of 5,803 patients with contrast exposures, 304 patients (5.2\%) had CIAKI. Patients in the statin group had an overall lower incidence of CIAKI (3.6\%) compared to the control group (6.9\%). Intravenous (IV) fluid hydration was used in both groups of all included studies for prevention of CIAKI. There was a significant protective effect of periprocedural statins on the incidence of CIAKI when compared to the control group [risk ratios (RR): 0.49 ; $95 \%$ CI: $0.37-0.66, \mathrm{I}^{2}$ of $\left.25 \%\right]$.

Conclusions: Our study demonstrates a statistically significant protective effect of statin treatment during procedures with contrast exposures. This finding suggests the use of statins in addition to standard IV crystalloid hydration may be beneficial in the prevention of CIAKI.

Keywords: Contrast-induced acute kidney injury, Metaanalysis, Statins.

11

\section{A Rare Case of Atypical Hemolytic Uremic Syndrome Associated with a Complement Factor H Gene Mutation in a Young Child Undergoing Induction Chemotherapy for Acute Lymphoblastic Leukemia}

\section{A. Pal, K.J. Reidy, A. Sinha, C.E. Straatmann \\ Pediatric Nephrology, Montefiore Medical Center, Bronx, NY, USA}

A three-year-old female diagnosed with precursor B cell acute lymphoblastic leukemia presented to the oncology clinic with gross hematuria, oliguria and blood tinged watery diarrhea. She had recently started on induction chemotherapy, including weekly vincristine. Physical examination was significant for new onset hypertension and weight gain. She was admitted and noted to be anuric. Laboratory findings revealed worsening anemia, thrombocytopenia, and elevated blood urea nitrogen, serum creatinine and

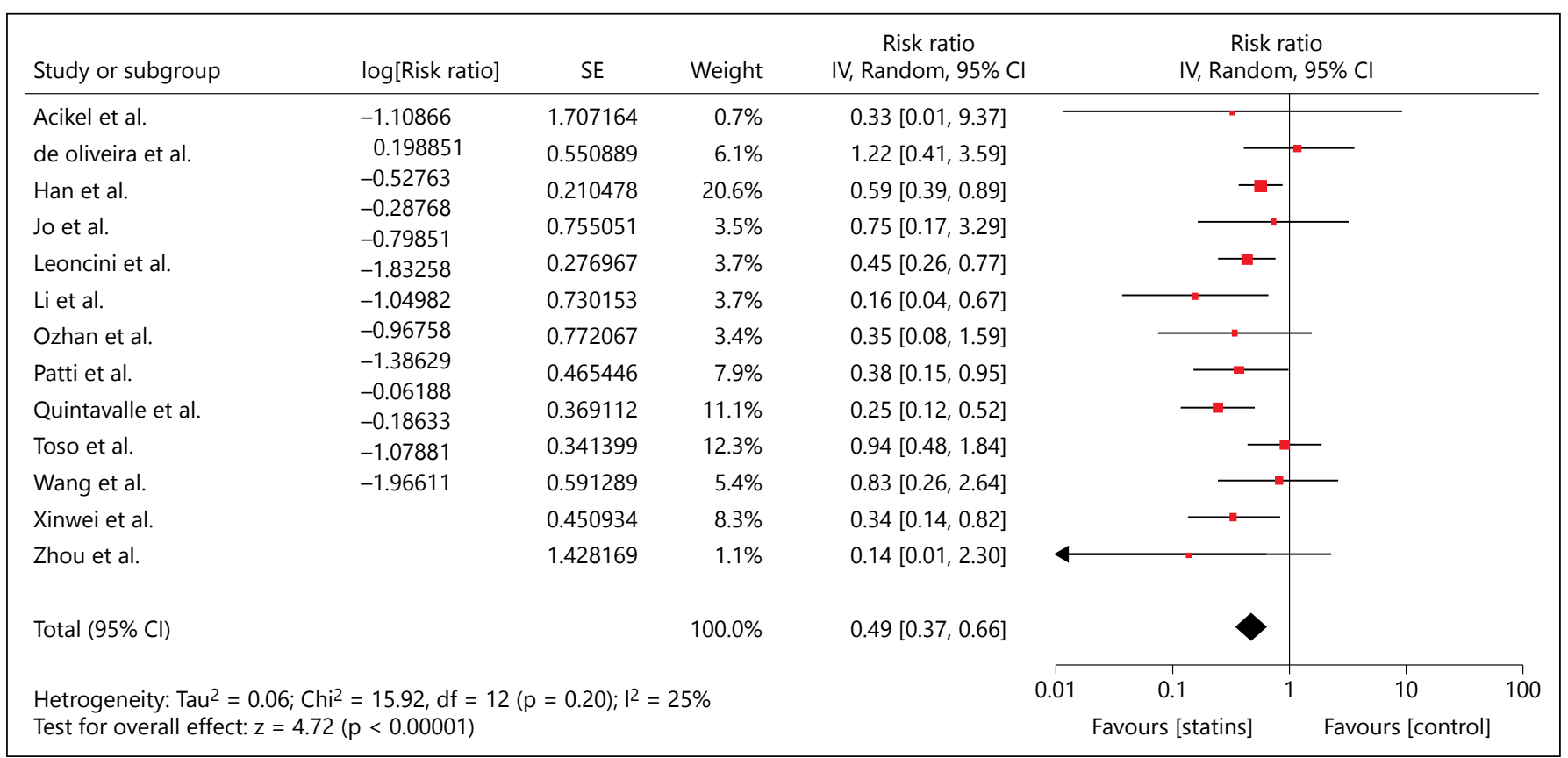

Fig. 1. (for Abstract 10). 
hyperkalemia. Schistocytes were present on a peripheral blood smear, consistent with microangiopathic hemolysis. She was diagnosed with atypical hemolytic uremic syndrome (aHUS). Stool culture was negative for Escherichia coli O157:H7. She received four treatments of plasmapheresis and two cycles of acute intermittent hemodialysis with resolution of her symptoms and normalization of laboratory values. Genetic testing revealed a heterozygous missense mutation in exon 19 (NM_000186: c. $2850 \mathrm{G}>\mathrm{T}$, p.Gln950His) of the complement factor $\overline{\mathrm{H}}(\mathrm{CFH})$ gene. This CFH mutation was previously reported in cases with sporadic aHUS. The patient continued to receive vincristine and her leukemia went into remission. After $11 / 2$ years of follow-up, she has no evidence of aHUS recurrence.

Prior reports of aHUS in association with malignancies, treatment with vincristine and after stem cell transplantation, have not included genetic testing. Our case report suggests that genetic predisposition may contribute to development of aHUS, even in the setting of exposure to known triggers of disease. Genetic testing can be considered in these cases.

Keywords: Hemolytic uremic syndrome, Acute lymphoblastic leukemia, $\mathrm{CFH}$ mutation.

12

\section{Incidence of Contrast-Induced Nephropathy (CIN) after Contrast-Enhanced CT Scan in the Inpatient Children}

M.C. Isaza López' , L.F. Rojas Rosas ${ }^{2}$

${ }^{1}$ Pediatric Resident, CES University, Medellín, Colombia;

2Pediatric Nephrology, Medellín General Hospital, Medellín,

Colombia

Background: The reported incidence in children of CIN is 4.8$10.6 \%$. We aimed to determine the incidence of CIN after contrast-enhanced CT.

Methods: Prospective and descriptive study. We enrolled patients under 14 years old who received intravenous hypo-osmolar nonionic iodinated contrast media (CM) for CT. CIN was defined as an increase in serum creatinine $>25 \% 48$ to 72 hours after contrast administraion. Patients were excluded if they were exposed to a CM 15 days before. The study is being conducted at two Hospital's Pediatrics Departments since January to December of 2014.

Results: Out of 230 subjects undergoing CT scans, 110 have been included. Among them, CIN incidence was 18\% (20/110). 58 were male, the mean age was 85.5 months. $50 \%$ of CT scan were indicated in the diagnostic study of abdominal infections. CM dose in $\mathrm{mg}$ Iodine $/ \mathrm{kg}$ was higher in CIN (median dose $396 \mathrm{mg} / \mathrm{kg}$ vs $312 \mathrm{mg} / \mathrm{kg}$ ). Some patients received intravenous hydratation but that was not part of an established nephroprotection protocol. According to RIFLE, 2 patients had increased risk, 4 had renal injury and 2 had failure; 4 of whom developed CIN. 13 out of 20 patients of CIN had anemia, however, our study group's overall anemia prevalence was $50 \%$. 3/5 patients with pharmacologic hemodynamic support developed CIN but $30 \%$ of patients with CIN were at PICU. Dipyrone-metamizol was the nephrotoxic drug most common used (57 patients), followed by vancomycine and aminoglycosides. The use of nephrotoxic drugs in CIN patients was 55\%. One patient received N-acetylcysteine for RIFLE F and he didn't develop CIN. One patient needed dialysis therapy but he also had a tumor lysis syndrome at the time. There were no deaths.

Conclusions: The high incidence of CIN (20\%) would suggest the need for implementing nephroprotection protocols and a closer follow up of the contrast-media exposed children.

The CM dose was higher in the CIN patients.

The use of nephrotoxic drugs and anemia are prevalent in our pediatric inpatient population.

Keywords: Contrast-induced nephropathy, Acute kidney injury, Contrast media.

\section{3 \\ Neurologic Symptoms in Non-Oliguric Hemolytic Uremic Syndrome}

\section{B. Crawford, D. Selewski}

University of Michigan Health System, Ann Arbor, MI, USA

Introduction: Hemolytic uremic syndrome (HUS) is characterized by the classic triad of hemolytic anemia, thrombocytopenia, and acute kidney injury (AKI). Neurological symptoms appear in approximately $25 \%$ of patients with HUS and have significant morbidity and mortality. The following case described neurological findings amidst non-oliguric AKI.

Case Report: An otherwise healthy 7 year-old girl presented with five days bloody diarrhea and stool positive for E. coli Shiga Toxin. On presentation, she was non-oliguric, with last urine void two hours prior to presentation. Laboratory studies were consistent with classic triad of azotemia, hemolytic anemia, and thrombocytopenia. Following admission, she received continuous intravenous fluid hydration with serial laboratory monitoring. She remained non-oliguric. Around 24 hours following admission, she developed acute neurological findings with left-sided facial droop and left-sided arm weakness. Emergent head imaging, blood pressure monitoring, and serum electrolyte measurement were all normal. Her symptoms self-resolved within 30 minutes, and she returned to her neurological baseline. Following this episode, she underwent three days of total plasma exchange (plasmapheresis). There were no further episodes of focal weakness during her course.

Discussion: This case highlights the importance of monitoring for neurological symptoms in all patients with HUS. Additionally, this case also highlights the use of total plasma exchange (plasmapheresis) for HUS-associated neurological findings, although additional research is needed.

Keywords: Hemolytic uremic syndrome, Neurologic symptoms, Plasmapheresis. 


\section{Peritoneal Dialysis}

14

\section{Acute Peritoneal Dialysis In African Pediatric Area Experience of Pediatric Unit Nephrology of Yopougon University Hospital (abidjan, Cote d'Ivoire)}

G. Diarrassouba, L. Adonis-Koffy, E. Niamien, J. Yaokreh

University Hospital, Abidjan, Côte d'Ivoire

Background: The acute renal failure (ARF) is the reduction or cessation of glomerular filtration. This is a diagnostic emergency may lead to fluid and electrolyte disorders involving life-threatening. The choice for the method of extra-renal expurgation in pediatric is limited in our african countries. The peritoneal dialysis could be the most practical modality for the children who have the acute renal failure.

Methods: We have doing a retrospective study on 5 years. The Children's files that have 1 month to 15 years and have the acute renal failure treated in Pediatric Nephrology Unit of Yopougon Academic Hospital and profit a peritoneal dialysis in acute moment. The definition of acute renal failure has been for the children a plasmatic creatinin most or equal than $200 \mu \mathrm{mol} / \mathrm{l}$, except the malformative kidney's diseases.

Results: During the study moment, 88 children have been hospitalized for acute renal failure with the indications of peritoneal dialysis, for 33 children. 22 children have been dialyzed with 9 retirements for absence of money. The sex ratio was $0.46(7 / 15)$. The middle age is 8,1 years with the extreme of 2 at 15 years. The reasons were dominated by the glomerular diseases (45\%), malaria $(31,8 \%)$ and the secondary interstitial nephritis caused by toxics drugs. The indications of peritoneal dialysis were represented by absence of kidney's water (31\%), hyperkalemia (13\%). The technics used were the peritoneal dialysis automatized for 8 children, the manual peritoneal dialysis for 2 children and for 9 children the both were used. 17 patients on the 22 have presented the complications as 31 mechanics complications and 10 infectious. We note $36,6 \%$ of death with $45,45 \%$ of recoving of kidney function.

Conclusions: In the end of study, the success of peritoneal dialysis automatized reposed on the efficient formation of medical and surgical agents and the better life of patients. The correct treatment of tonsillitis and effective management of malaria should reduce the occurrence of the ARF.

Keywords: Peritoneal dialysis, Children, Africa.
15

\section{End-Stage Kidney Disease and Hepatoblastoma:} Three pediatric patients at a single center

\section{A. Kouri, J.M. Misurac, A.C. Wilson}

Nephrology, Riley Hospital at Indiana University, Indianapolis, IN, USA

Background: Hepatoblastoma is the most common pediatric hepatic malignancy. Nonetheless, it is very rare with an estimated incidence of 1.6 per 1 million children under the age of 19 years.

Methods: Retrospective case series.

Results: In the last four years at our center, three patients with end stage renal disease (ESRD) secondary to renal dysplasia have presented with hepatoblastoma. All patients were on dialysis since the neonatal period. The first patient was a 2.5 year old male with bilateral renal dysplasia on hemodialysis. Shortly after his family moved out of state, liver masses were incidentally discovered on renal ultrasound and found to be hepatoblastoma. He received chemotherapy but his cancer recurred; he is currently undergoing cisplatin/doxorubicin-based chemotherapy. The other two patients were both males with ESRD due to posterior urethral valves and dysplasia managed on peritoneal dialysis. The second patient presented at 4.5 years of age with abdominal pain and constipation. Abdominal ultrasound revealed a large liver mass, which proved to be stage III hepatoblastoma. He received cisplatin/doxorubicin based chemotherapy prior to a combined liver/kidney transplant. The third patient was a 23 month old in whom asymptomatic liver masses were found on routine renal ultrasound; liver biopsy revealed hepatoblastoma. He is undergoing cisplatin/doxorubicin-based chemotherapy.

Conclusions: The approximate incidence of hepatoblastoma at our center over the past 4 years was 3.5\% (3/84). Further study is indicated to determine if there is a true association between renal dysplasia and hepatoblastoma.

Keywords: Hepatoblastoma.

\section{6}

\section{Lymphangiogenesis and Lymphatic Absorption are Related and Increased in Chronic Kidney Failure, Independent of Dialysis Solutions}

\author{
Carmen A. Vlahu' ${ }^{1}$, Marijke de Graaf', Jan Aten ${ }^{2}$, Dirk G. Struijk ${ }^{1,3}$, \\ Raymond T. Krediet ${ }^{1}$
}

'Division of Nephrology, Department of Medicine, Academic Medical Center, Amsterdam, The Netherlands; ${ }^{2}$ Department of Pathology, Academic Medical Center, Amsterdam, The Netherlands; ${ }^{3}$ Dianet Foundation, Amsterdam-Utrecht, The Netherlands

Background: Increased lymphatic absorption may contribute to the development of ultrafiltration failure in PD. Lymphangiogenesis occurs during treatment with peritoneal dialysis but little is known about the relationship between the morphological and functional parameters.

Aim: The possible relation between the number of lymph vessels and the effective lymphatic absorption rate (ELAR) was inves- 
tigated in a model of rats with chronic kidney failure exposed to dialysis solutions.

Methods: Fourty-four Wistar rats were divided in four groups: NKF (normal kidney function), CKD (chronic kidney failure induced by $70 \%$ nephrectomy), CKDP (CKD receiving daily peritoneal infusions with Physioneal 3.86\%) and CKDD (CKD receiving daily peritoneal infusions with Dianeal $4.25 \%$ ). After 16 weeks a standard peritoneal permeability analysis adapted for rats (SPARa) was performed in rats from all groups and the ELAR was calculated from the disappearance of intraperitoneally administered dextran 70. Lymph vessels in omentum were visualized using anti-podoplanin immunostaining. STEPanizer image analysis was applied to assess lymph vessel profile density.

Results: The ELAR was lowest in NKF (12 $\pm 3 \mu \mathrm{l} / \mathrm{min})$, somewhat higher in CKD $(19 \pm 2 \mu \mathrm{l} / \mathrm{min})$, and highest in the exposed rats $(23.2 \pm 9.3 \mu \mathrm{l} / \mathrm{min}$ in CKDP and $29.2 \pm 22.1 \mu \mathrm{l} / \mathrm{min}$ in CKDD group) but the differences were not significant. The lymph vessel profile densities were: $0.3 \pm 0.2$ in NKF, $0.8 \pm 0.4$ in CKD, $0.9 \pm 0.4$ in CKDP and $1.0 \pm 0.6$ profiles/ $\mathrm{mm}^{2}$ in CKDD ( $\mathrm{p}<0.05$ for NKF vs all others). No difference in ELAR or lymphatic density was present between the groups exposed to two different dialysis solutions. For all rats together a positive correlation was present between the lymph vessel profile density and the effective lymphatic absorption rate: $\mathrm{p}=0.04$, Spearman's rho $=0.63$.

Conclusions: Chronic kidney failure itself induces lymphangiogenesis and increases the effective lymphatic absorption rate. Exposure to dialysis fluids does not have a significant additional effect.

\section{Pediatric Nephrology}

17

The Prevention of Children Kidney's Diseases by Using the Urinary Bandelet During a School Medical Visit. Case of Hematobium Schistosoma Disease

\section{Adonis-Koffy, G. Diarrassouba \\ University Hospital, Abidjan, Côte d'Ivoire}

Background: Our study has aim to evaluate the efficiency of macroscopic exam of urine by the urinary reactive bandelet in detection of hematobium schistosoma in school in the health district of Yamoussoukro during the school medical visit.

Methods: It was a analytic and descriptive prospective study in 13 primaries schools during 6 months. Yamoussoukro is the political and administrative capital of the COTE D'IVOIRE. Schools are located in village's private health facilities and easy access to the study. The students included in the study are those who presented a antecedent of blood urine, a blood urine and or a protein in urine at the urinary bandelet and who has beneficed a parasitological exam of urine.

Results: On 2014 students, 433 has been included and 319 were infected by hematobium schistosoma, a proportion of $15,8 \%$ of urinary schistosoma in the locality and of $74 \%$ between the students. The sex ratio was 1, 6 with the middle age of 10 years. The swimming in stagnating water $(85,5 \%)$, the frequentation of irrigated cultures $(78,8 \%)$, the missing of good water $(90,53 \%)$ are the factors incriminated in the transmission of disease.The urinary bandelet has showed for the blood urine a sensibility of 96 , $8 \%$, a positive predictive value of $83,6 \%$ and a negative predictive value of $84,16 \%$ for the schistosoma. For the protein in urine, the bandelet has a good sensibility of $85,8 \%$, a positive predictive value of $52,12 \%$.

Conclusions: The study shows the importance of systematic school medicals visits. The research of blood urine and protein in urine with aid of bandelet rests a simple and efficient mean of detection of schistosoma and prevention of kidneys diseases in our poor's countries.

Keywords: Schistosoma, School medical visit, Urinary bandelet.

\section{8}

\section{Prevalence of Nocturnal Hypertension and Nocturnal Dipping of Blood Pressure in Pediatric Kidney Transplant Recipients}

\author{
M. Almonte' ${ }^{1}$ S. Enciso ${ }^{2}$, A. Hernandez 3 , L. Ortiz' ${ }^{4}$ H. Gonzalez', \\ M. Medeiros ${ }^{1}$
}

'Pediatrics Nephrology, Hospital infantil de México Federico Gómez, México city, Mexico; ${ }^{2}$ nephrology research laboratory and bone and mineral metabolism, Hospital infantil de México Federico Gómez, México city, Mexico; ${ }^{3}$ nephrology reseacrh laboratory bone and mineral metabolism, Hospital infantil de México Federico Gómez, México city, Mexico; ${ }^{4}$ Nephrology research laboratory and bone and mineral metabolism, Hospital infantil de México Federico Gómez, México city, Mexico

Introduction: Hypertension HT is a common complication in children and adolescents undergoing renal transplantation with a prevalence between $70-80 \%$. HT is linked to accelerated graft loss increased cardiovascular risk coronary artery disease left ventricular hypertrophy and death. HT can have multiple etiologies remaining native kidney medications graft dysfunction obesity and pre-existing vascular disease.

Objective: To determine the prevalence of nocturnal hypertension and nocturnal dipping of blood pressure in Mexican children with kidney transplants using ambulatory blood pressure monitoring.

Material and Methods: Cross-sectional study We included pediatric transplant patients within 3-12 months after renal transplant and stable graft function they all signed informed consent We excluded patients with prednisone $>0.5 \mathrm{mg} / \mathrm{kg} /$ day ABPM was performed for $24 \mathrm{~h}$ with an oscillometric device spacelabs model 90217-1a blood pressure was measured every 30 min during the day every hour during the night.

Results: Eighteen patients were included Nine had nocturnal hypertension None of the patients in the hypertension group showed nocturnal dipping of blood pressure, and dipping was observed in only three patients from the normotensive group Five patients in the normal blood pressure group $27.7 \%$ had history of graft rejection successfully treated and none in the hypertension group $\mathrm{p}=0.02$ There was no difference in renal function tacrolimus trough levels serum cholesterol serum triglycerides hepatic 


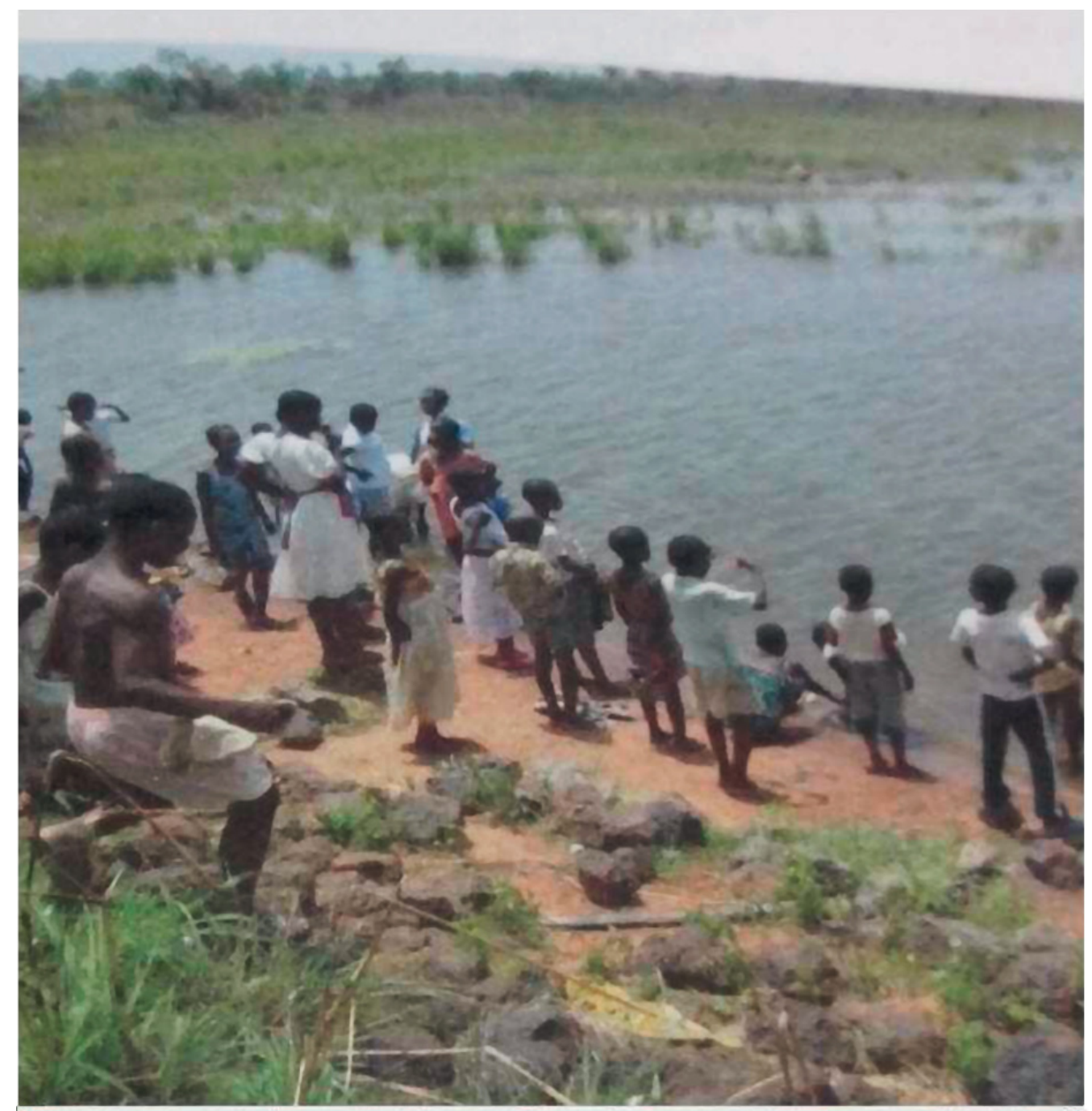

\section{THE PUPILS SWIMMING IN A LAKE OF YAMOUSSOKRO}

Fig. 1. (for Abstract 17).

enzymes hemoglobin and proteinuriaThere was no significant difference in glomerular filtration rate between the patients with nocturnal dipping and no dipping.

Conclusions: $50 \%$ of the patients with kidney transplant showed nocturnal hypertension detected by ABPM Normotensive patients had a higher number of previous graft rejection and probably a closer follow up with adjustment of anti-hypertensive medications Only $3 / 18$ had nocturnal dipping HT is a major problem in patients with kidney transplant ABPM allows identify patients with nocturnal HT.

Keywords: m, a, p.

\section{9 \\ Pediatric Dialysis Patient Charges: Decreasing Errors and Reclaiming Lost Revenue}

J.M. Misurac ${ }^{1}$, P.C. Froehlich ${ }^{2}$, J.K. Kansal ${ }^{3}$, K.W. Everly ${ }^{2}$, J.D. Leiser ${ }^{1}$

${ }^{1}$ Pediatric Nephrology, Riley Hospital for Children at Indiana University, Indianapolis, IN, USA; ${ }^{2}$ Kelley School of Business, Indianapolis, IN, USA ${ }^{3}$ Department of Surgery, University of Cincinnati, Cincinnati, OH, USA

Background: We addressed pediatric dialysis charge errors using the Six Sigma DMAIC process (Define, Measure, Analyze, Improve, and Control).

1. Define: A paper worksheet of daily charges is generated. Each charge is then entered electronically for each patient; this 
takes 9 clicks (about 30-60 seconds) per patient. There is no onscreen confirmation after a charge is entered.

\section{Methods:}

2. Measure: The paper charge sheet was compared with electronic billing history over the preceding 13 months. Three common errors were identified: a) Duplicate charges, which are automatically corrected, b) Incorrect charges, which could result in lost revenue or overbilling, and c) Missed charges, which represent a critical error resulting in lost revenue and unrecovered expenses.

\section{Results:}

3. Analyze: There were 227 errors in 9,664 charges, representing an error rate of $2.3 \%$. There were 99 duplicate charges, 32 incorrect charges, and 96 missed charges. Totaled, this represented $\$ 142,350$ in lost revenue.

4. Improve: We created a semi-automated AutoHotkey; this reduced the number of clicks for charge entry from 9 to 3 . We also developed an Excel ${ }^{\circledR}$ spreadsheet for monthly audits to compare paper charges with electronic billing.

\section{Conclusions:}

5. Control: The AutoHotkey was seen as a significant improvement by users and has been implemented. The monthly audits have not been performed as designed by our improvement team due to lack of personnel and time. However, we anticipate they will be fully implemented by new staff soon to be hired.

Keywords: Quality improvement, Dialysis, Billing.

\section{0}

\section{Cisplatin Nephrotoxicity in Children with Solid Tumors}

C.A. Jiménez-Triana ${ }^{1}$, D. Castelan ${ }^{1}$, R. Rivas-Ruíz', R. Jiménez-Méndez ${ }^{3}$, A. Medina ${ }^{1}$, B. Carleton ${ }^{3}$, R. Rassekh ${ }^{3}$, M. Medeiros-Domingo $0^{2,3}$

${ }^{1}$ Hospital Infantil de México, México DF, Mexico; ${ }^{2}$ Laboratorio de Investigación en Nefrología, Hospital Infantil de Mexico, Mexico DF, Mexico; ${ }^{3} \mathrm{BC}$ Children's Hospital, Vancouver, BC, Canada

Background: Cisplatin, a major antineoplastic drug used in the treatment of solid tumors is a known nephrotoxic. This study evaluates the prevalence and severity of cisplatin nephrotoxicity in children and its impact on height and weight.

Methods: Retrospective study of 54 cisplatin treated children. The weight, height, serum creatinine and electrolytes in each cisplatin cycle and after 12 months of treatment was recorded. Nephrotoxicity was graded as follows, 0 : normal renal function, 1 : asymptomatic electrolytes disorders, grade 2 : need for electrolyte supplementation $<3$ months and/or increase in serum creatinine 1.5-1.9 times from baseline, grade 3 : increase in serum creatinine 2-2.9 times from baseline or need for electrolyte supplementation for more than 3 months after treatment completion, grade 4: renal replacement therapy.

Results: Nephotoxicity (NTX) was observed in 41 patients (76\%). Grade 1 NTX was observed in 18, Grade 2 in 5 patients and Grade 3 in 18 patients. None required renal replacement therapy (grade 4). NTX patients were younger than patients with nonNTX ( $p=0.001)$, received higher cisplatin total dose (mean accu- mulated dose in NTX $455 \mathrm{mg} / \mathrm{m}^{2}$ vs. $372 \mathrm{mg} / \mathrm{m}^{2}$ in non-NTX, $\mathrm{p}=$ 0.01 ), and had more diverse tumors than non-NTX patients in whom germinal tumors were more frequent. Patients without NTX maintained their $\mathrm{z}$ Score for height after 12 months of treatment, whereas those with NTX had worse $\mathrm{z}$ Score for height after 12 months of treatment, being more accentuated in those with NTX grade 1 than grades 2 and 3 . There was no difference in $z$ Score in weight (basal vs. 12 months) in all groups. The cisplatin total dose had a significant negative relationship with magnesium levels at 12 months.

Conclusions: NTX prevalence was 76\%. NTX patients were younger and received higher cisplatin doses than those with normal renal function. Patients with NTX had a significantly worse height $\mathrm{z}$ Score at 12 months than those with normal renal function, patients with untreated electrolyte anomalies (NTX grade 1) were the most affected. Cisplatin total dose has a negative correlation with magnesium serum levels at 12 months. Patients receiving cisplatin should be monitored during each cycle of treatment for evidence of grade 1-4 nephrotoxicity. Even asymptomatic electrolyte abnormalities can cause clinically significant effects on height.

Keywords: Cisplatin toxicity, Electrolyte disorders, Growth.

\section{1 \\ Renal Tubular Dysgenesis: An unusual Cause of Neonatal Renal Failure}

\section{R.S. Zahr, R. George, R. Garro}

Pediatric Nephrology, Emory School of Medicine, Atlanta, GA, USA

In the newborn period approximately $97 \%$ of neonates will void within the first 24 hours and nearly all will void within 48 hours. The differential diagnosis of neonatal oligonauria can be divided into 3 categories primary non-renal disease, primary renal disease and post renal failure. A less common reason that must be considered is renal tubular dysgenesis (RTD). The histological hallmark is the absence or poor development of renal proximal tubules. Except for a very few cases, the prognosis has been poor secondary to renal failure, severe oligohydraminos, and pulmonary hypoplasia leading to fetal demise. RTD occurs due to autosomal recessive inheritance or is secondarily acquired. Here we report a case of an infant born at 40 weeks, birth weight of $3.02 \mathrm{~kg}$, without history of oligohydraminos or pulmonary hypoplasia who was diagnosed histologically with RTD. He was delivered via cesarean section secondary to fetal distress and intubated for respiratory depression due to meconium aspiration. In the NICU he was quickly extubated after 1 dose of antibiotics. After 24 hours without voiding, labs were drawn and notable for creatinine of $3.9 \mathrm{mg} / \mathrm{dl}$. Renal ultrasound showed normal sized echogenic kidneys without urine in the bladder. Infant remained anuric with progressive anasarca and electrolyte derangements. A peritoneal dialysis catheter was placed after one week of age and renal biopsy performed at two weeks of age was consistent with RTD. The diagnosis of RTD should be considered in any anuric infants with normal kidney size and structure on ultrasound. This case is unique as our patient is now 9 months old and remains maintained on peritoneal dialysis with hopes to transplant.

Keywords: Renal tubular dysgenesis, Renal failure. 


\section{2}

\section{State Level Variations in Nephrology Workforce and Timing and Incidence of Dialysis in the United States Among Children and Adults}

\author{
Elaine $K \mathrm{u}^{1,2}$, Kirsten L. Johansen ${ }^{1,3,4}$, Anthony A. Portale ${ }^{2}$, \\ Barbara Grimes ${ }^{3,4}$, Chi-yuan Hsu ${ }^{1,4}$ \\ ${ }^{1}$ Division of Nephrology, Department of Medicine, ${ }^{2}$ Division of \\ Pediatric Nephrology, Department of Pediatrics, ${ }^{3}$ Department \\ of Biostatistics and Epidemiology, ${ }^{4}$ United States Renal Data \\ System, Nutrition Special Studies Center, University of \\ California, San Francisco, CA, USA
}

Background: Multiple factors influence timing of dialysis initiation. The impact of systems-based factors such as supply of nephrology workforce on timing and incidence of dialysis initiation is not well known.

Methods: We determined the number of pediatric and adult nephrologists in each state using data from the American Medical Association and American Boards of Internal Medicine and Pediatrics. We ascertained state population data from the 2010 US Census. United States Renal Data System (USRDS) data were used to determine estimated glomerular filtration rate (eGFR) at dialysis initiation and dialysis incidence for adults ( $\geq 18$ years) in 2008 and children ( $<18$ years) in $2007-2009$ by state.

Results: Across all states, there were a median of 3.0 (IQR 2.3 to 3.4) adult nephrologists per 100,000 adults and 0.5 (IQR 0.3 to $0.9)$ pediatric nephrologists per 100,000 children. The median eGFR at start of dialysis was $9.8 \mathrm{ml} / \mathrm{min} / 1.73 \mathrm{~m}^{2}$ (IQR 7.1-13.1) in adults and $8.5 \mathrm{ml} / \mathrm{min} / 1.73 \mathrm{~m}^{2}$ (IQR 6.2-11.4) in children. Neither the number of adult (Spearman $\mathrm{r}=0.02 ; \mathrm{p}=0.88$ ) nor pediatric (Spearman $\mathrm{r}$ of $-0.13 ; \mathrm{p}=0.38$ ) nephrologists/state population was associated with mean eGFR across states. The number of nephrologists/state population was associated with incident dialysis cases per state in adults (Spearman $\mathrm{r}$ of $0.50, \mathrm{p}=0.002$ ), but not in children (Spearman $r$ of $-0.06, p=0.67$ ). Similar results were seen in alternative analysis using board certification data to ascertain physician workforce.

Conclusions: Nephrology workforce supply is aligned with demand but does not appear to be associated with timing of dialysis initiation.

Keywords: Nephrology workforce, Timing of dialysis.

\section{3 \\ The STARx Survey as an Auxiliary to the TRxANSITION scale in Measuring Healthcare Transition Readiness}

\section{P.J. Leahy, J.S. Karina, S.E. Cohen, M.E. Ferris}

Pediatric Nephrology, University of North Carolina, Chapel Hill, NC, USA

Background: There is a need for methodology to appropriately assess readiness for transition to self care among young adults. One such method called the UNC TRxANSITION Scale has previously been described. An important adjunct to the TRxANSITION Scale is the self-administered STARx Survey. The
STARx Survey has not been correlated to the following tools: TRxANSITION Scale, Morisky Medication Adherence Scale or the Peds Quality of Life (PedsQoL) in the young adult or pediatric population.

Methods: Patients (ages 12-29) seen in the pediatric- or adultfocused settings between 2008 and 2013 participated. Their medical conditions included CKD/ESRD $(n=46)$, IBD $(n=29)$, cystic fibrosis $(n=12)$. Participants with SLE, sickle cell disease, hypertension and HIV were also recruited. The six STARx subdomains were compared to all other scales with 2-tailed Pearson tests.

Results: Of 194 patients (52.1\% males, 55.7\% white; $28.9 \%$ AA; and $4 \%$ public insurance), all completed the STARx Survey, 139 completed the TRxANSITION Scale; 57 completed the PedsQoL, and 139 completed the Morisky Medication Adherence Scale. The STARx Survey and six subdomains correlated with the TRxANSITION Scale and Morisky Medication Adherence Scale at various levels but did not correlate to the PedsQoL.

Conclusions: The significant relationship between the selfadministered STARx Survey and TRxANSITION Scale provides evidence for their use in the clinical setting. However, the STARx Survey did not predict quality of life.

Keywords: Transition, STARx, Pediatric nephrology.

\section{4}

\section{Learning Platform and Kidney Knowledge among Adolescents with CKD}

\section{A. Phillips, S. Cohen, A. Annaim, M. Ferris}

UNC Kidney Center, University of North Carolina at Chapel Hill, Chapel Hill, NC, USA

Background: With the growing availability and access to the Internet, the way pediatric patients learn about their chronic conditions may be likely to change from handouts and brochures to online sources of information. Changes in learning styles could have a substantial impact in the knowledge patients have of their conditions.

Methods: English-speaking patients aged 10 and older with a diagnosis of CKD stage II or greater were recruited in a pediatric nephrology clinic. Patients were administered the Kidney Knowledge (Kikks) survey (Wright et al. 2001), a 14 question multiple-choice assessment of knowledge about kidney disease. In order to discern their preferred learning platform, patients were provided a list of possible platforms, from which they chose the ones they preferred.

Results: We enrolled 32 patients with the following characteristics: mean age was $15.03( \pm 2.37)$; female (58.1\%); AfricanAmerican 33\%, Caucasian 29\%, Hispanic 29\%; and public insurance $48 \%$. Participants' favorite sources of information were their doctor/nurse $(77.4 \%)$, their parents $(48.4 \%)$, and the internet (45.2\%).

The average performance for the kidney knowledge survey was $8.45( \pm 2.65)$ out of 13 . Participants who reported a higher frequency of learning from parents and family also performed better on the knowledge survey $(r=0.392, p=0.03)$.

Conclusions: Pediatric patients with Chronic Kidney Disease do not seem to prefer the internet for information over their doctors or parents, but do prefer it over booklets or brochures. 
Patients who report learning more from parent and family also know more about their condition. With this knowledge, patients should be encouraged to learn from trusted sources of information (parents, doctors) in order to ensure good knowledge of their condition.

Keywords: Knowledge, Learning, Chronic kidney disease.

25

\section{Parental Knowledge of CKD in Relation to Youths' Knowledge}

A. Annaim, S. Cohen, A. Phillips, M. Ferris

University of North Carolina-Chapel Hill, Chapel Hill, NC, USA

Background: Chronic Kidney Disease (CKD) is a condition that places a large degree of responsibility on patients and their parents because of the necessity to manage several medications, and comply with nutrition needs. Pediatric patients depend for proper medical care.

Methods: We performed the Kidney Knowledge survey with pairs of parents and children where the children had a diagnosis of at least Stage II CKD at the tertiary center's Nephrology clinic. We enrolled 18 pairs of parents and children.

Results: We enrolled 18 parents (two fathers and 16 mothers). The mean age for the children was 15 years old. The mean percentage of life with disease was $52 \%$ ( $\mathrm{SD} \pm 8.9 \%$ ). $22 \%$ of the par- ents were Hispanic, $44 \%$ were Caucasian, and 33\% were African-American. $50 \%$ of the parents had private insurance.

Parents average score had a weak correlation with youth score when controlling for insurance status, race, and the child's percentage of life with the disease $(\beta=-0.045, p=0.86)$. This analysis was done via a multivariable linear regression model.

Conclusions: Parental knowledge of CKD does not appear to have correlate well with youth knowledge when controlling for potential confounders. Parental knowledge of CKD is likely dependent on many factors. Identifying these factors through future research can provide areas for intervention, which may lead to potential improvements.

Keywords: Parent knowledge, Chronic kidney disease.

26

\section{Transient Thrombotic Microangiopathy Following Living Related Kidney Transplantation}

N. Herrera' ${ }^{1}$, E. Sanchez ${ }^{2}$, B. Vogt ${ }^{1}$

${ }^{1}$ Pediatric Nephrology, University Hospital's Rainbow Babies \& Children's Hospital, Cleveland, OH, USA; ${ }^{2}$ Surgery, University Hospital's Rainbow Babies \& Children's Hospital, Cleveland, $\mathrm{OH}$, USA

Background: Thrombotic microangiopathy (TMA) is a histopathological description of vessel wall thickening, intraluminal
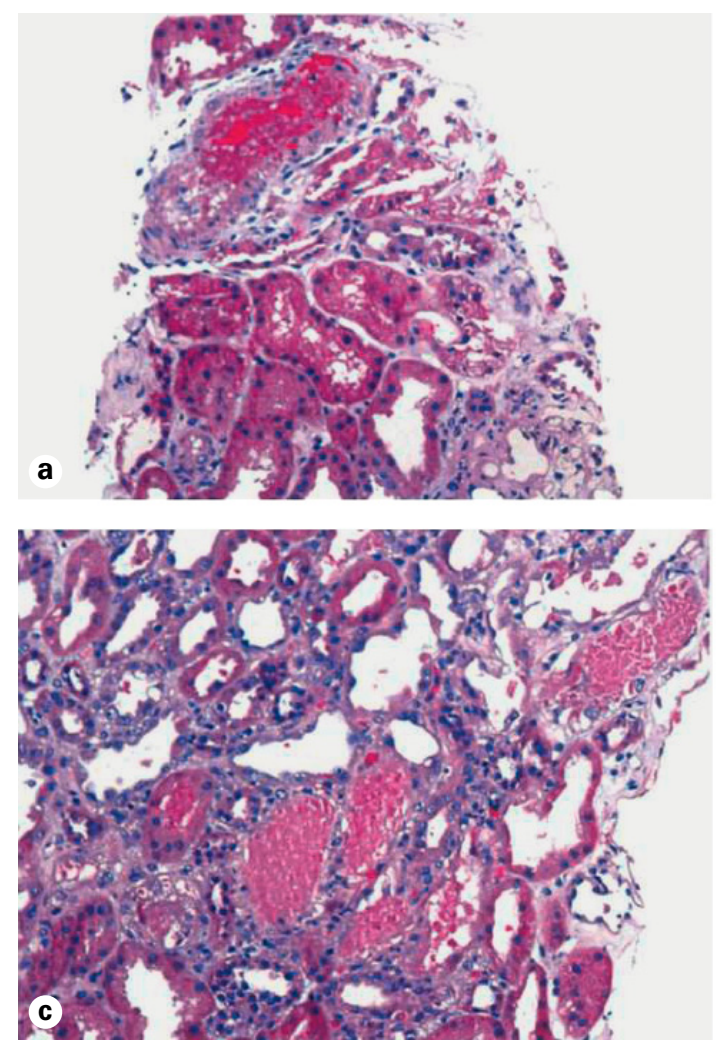
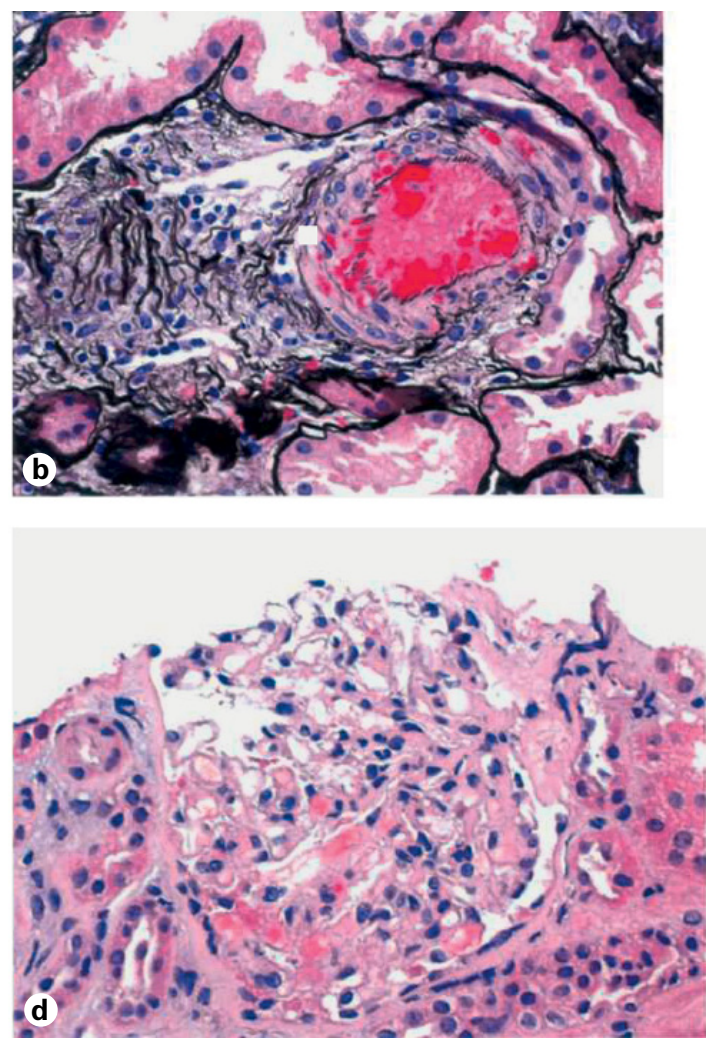

Fig. 1. (for Abstract 26). 
platelet thrombosis, and arteriolar obstruction. Clinically it is associated with thrombocytopenia and microangiopathic hemolytic anemia. TMA can be a serious complication of kidney transplantation and may result in allograft failure. Most reports of TMA have been associated with calcineurin inhibitor, acute antibody-mediated rejection, or recurrence of atypical hemolytic uremic syndrome (aHUS). We present a child in whom transient TMA likely occurred related to unintentional prolonged surgical time.

Case: A 2 year old boy with CKD-5 from birth asphyxia underwent living related donor transplantation with a right kidney. He received basiliximab, mycophenolate mofetil, and methylprednisolone. Calcineurin inhibitor was held awaiting allograft function. After initial reperfusion, the allograft demonstrated significant outflow insufficiency that required removal, hypothermic re-cooling, and re-implantation (Total warm ischemia time 88 minutes). By post operative day 4, progressive hemolytic anemia, thrombocytopenia and delayed graft function prompted suspicion of TMA. Renal biopsy demonstrated TMA and acute tubular necrosis (figure 1.). Clinical features of TMA improved gradually and allograft function was established by day 10 .

Conclusions: TMA is a rare complication of renal transplantation, usually occurring in patients with previous history of aHUS or as an adverse effect of calcineurin therapy. TMA has also been reported following prolonged warm ischemia time, although this complication is usually described in deceased donor transplants or associated with antibody-mediated rejection. Our patient developed self-limited TMA following prolonged surgical time during living donor transplantation, presumably as a consequence of endothelial injury related to ischemia-reperfusion.

Keywords: Thrombotic microangiopathy, Hemolytic uremic syndrome, Renal transplant.

\section{Hemodialysis}

\section{7 \\ Tolerability and Resource Utilization in Dialysis Patients Undergoing Treatment for Tuberculosis Related Conditions}

A.M. Hamadah, L. Beaulieu, J.W. Wilson, T.R. Aksamit, J.R. Gregoire, A.W. Williams, J.J. Dillon, R. Albright, V.K. lyer, L.J. Hickson

Mayo Clinic, Rochester, MN, USA

Background: The incidence of tuberculosis (TB) in end stage renal disease (ESRD) is significantly higher than the general population. Treatment for TB-related conditions has been associated with increased side effects especially among those with kidney dysfunction. However, little is known of its impact in chronic hemodialysis patients.

Objective: To determine the frequency of side effects, tolerability, and pattern of resource utilization in hemodialysis patients receiving anti-TB therapy.

Methods: Case series of dialysis patients receiving anti-TB therapy between 2002-2014 at a single dialysis institution.
Results: A total of 12 patients were treated for Latent $(n=7)$ or Active $(n=5)$ TB. Median age at treatment was 60 (range 24-86). $75 \%$ were males; $42 \%$ were Caucasian. Cause of ESRD was diabetes in 50\%. Anti-TB therapy was administered for a median of 5.5 months. Anti-TB therapy for Latent TB patients consisted primarily of isoniazid monotherapy $(n=5)$ or rifampin based therapy $(n=2)$. Anti-TB therapy for Active TB patients was comprised of a four or more drug isoniazid-based regimen. The most frequent adverse effects of anti-TB therapy were lightheadedness (50\%), syncope (42\%), and liver enzyme elevation (25\%). Anti-TB treatment was modified due to symptoms in $33 \%$ of patients overall. A majority $(75 \%)$ were hospitalized (median visits 1 ; range $1-3$ ) or treated in the emergency department (25\%) (median visits 1; range 1-6) for complications or symptoms experienced during the treatment period.

Conclusions: Anti-TB therapy is associated with frequent side effects leading to increased health care resource utilization in chronic hemodialysis patients. Increased awareness of potential complications and careful monitoring of patients may be essential to optimize medication adherence and minimize hospitalization rates during this period.

Keywords: Tuberculosis, Dialysis, Treatment.

\section{8 \\ Calciphylaxis in Recent Hemodialysis: A Report of Two Cases}

\section{A. Sanguankeo, S. Upala, N. Thamcharoen, N. Leeaphorn, R. Rosen}

Bassett Medical Center and Columbia University College of Physicians and Surgeons, Cooperstown, NY, USA

Background: Calciphylaxis or calcific uremic arteriolopathy (CUA) is a rare complication of end stage renal disease (ESRD) patients particularly in dialysis dependent. This condition is also related to high morbidity and mortality. We report two cases of early ESRD dialysis-dependent patients with different presentation of calciphylaxis.

Methods: Case 1: A 65 year old woman had been diagnosed with diabetes, ESRD and dialysis dependent since 2013. She has history of hyperparathyroidism and hyperphosphatemia with baseline phosphorus of 8-9 mg/dl (2.5-4.9) and PTH of $421 \mathrm{pg} /$ $\mathrm{ml}(14-72)$ and receives sevelamer as treatment. She presented one year after starting hemodialysis with abnormal calcified phlebolith presenting in the anterior subcutaneous soft tissues of the lower left leg. Whole body bone scan revealed soft tissue uptake identified bilateral lower extremity. She was diagnosed with calciphylaxis and given sodium thiosulphate $25 \mathrm{mg}$ intravenously 2-3 times per month for 3 months with a follow up phosphorus range between 4.0-8.6.

Case 2: A 66 year old woman presented for evaluation of firm subcutaneous plaques present on her legs in November 2013. These have spread to involve almost her entire medial thigh, lateral thigh, hips, lateral knees, and proximal calves. Her medical history included diabetes, ESRD and secondary hyperparathyroidism with baseline PTH of 147 and normal calcium, phosphorus level. She began hemodialysis in September 2013. Physical examination revealed impressively firm non-confluent subcutaneous plate like plaques involving the hips, lateral and medial thighs. 
Biopsy from biopsy from the lesion on hip found fat necrosis with calcification within the subcutaneous adipose tissue, calcification of small caliber vessels of the deep plexus. Sodium thiosulfate was given 4 times per month for 4 months. Post-treatment phosphorus range between $4-5$.

Conclusions: Calciphylaxis mainly affects patients with late stage of ESRD and has been reported to have a prevalence of $4 \%$ in dialysis patients. Elevated Calcium, Phosphorus, and PTH levels, a high Ca-P product are important risk factors for calciphylaxis. The presence of concomitant diabetes also increases the risk of calciphylaxis and the development of acral gangrene. These cases were similar in that they recently started hemodialysis and had diabetes and hyperparathyroidism. However, the latter case had normal calcium and phosphate, which make them less likely a major contribution to calciphylaxis. This condition has poor prognosis and require multimodal therapeutic approach to increase survival.

Keywords: Calciphylaxis, Hemodialysis, Hyperphosphatemia.

\section{9}

Relationship between Carotid Artery Calcified Plaque and GA (Glycoalbumin), Serum Phosphorus Level in Dialysis Patients

\section{Y. Sawamura}

Sapporo Hokuyu Hospital, Sapporo, Japan

Background: A relationship with GA, serum phosphorus level calcified Plaque in Dialysis patient' Carotid Artery Ultrasound test.

Methods: Three carotid artery ultrasound tests were performed on 92 diabetic patients receiving dialysis (53 men, 39 women) and 86 non-diabetic patients receiving dialysis ( 47 men, 39 women) with six month intervals between tests. During each exam, IMT thickness was measured and the appearance of calcified plaques was noted. In 32 patients (8 diabetics and 24 nondiabetics), calcified plaques were observed during the third exam after the first two exams showed no evidence of calcified plaques. The thickness of the calcified plaques was measured (the sum of both sides). Over the course of a year, these 32 patients were examined every month and the percentage of HbA1c, NGSP value, GA percent, HDL, LDL, non-HDL, LDL/HDL ratio, blood pressure immediately before dialysis, weight gain between dialysis procedures, $\mathrm{Na}, \mathrm{K}, \mathrm{Ca}, \mathrm{HS}-\mathrm{PT} \mathrm{H}, \mathrm{P}, \mathrm{CaP}$ product, Alp, and $\beta 2 \mathrm{MG}$ were measured.

Results: (1) Serum phosphorus level of non diabetic 24 dialysis patients with new calcified plaque is $5.3 \pm 0.9 \mathrm{mg} / \mathrm{dl}$ (vs. $4.8 \pm$ $0.7 \mathrm{mg} / \mathrm{dl}, \mathrm{p}=0.028523$ no calcified plaque of non diabetic dialysis patients in this study).

(2) In diabetic dialysis patients, A newly appeared thickness of the calcified plaque (sum of the total value of right and left sides) correlation was accepted by the GA (\%) and following formula. $\mathrm{y}=0.0902 \mathrm{x}+1.203 \mathrm{p}=0.0298 \mathrm{R}=0.60$ in 8 diabetic dialysis patients.

Conclusions: (1) Serum phosphorus level is thought that it is desirable less than $5.0 \mathrm{mg} / \mathrm{dl}$ in non diabetic dialysis patients.

(2) In the diabetic dialysis patient, the sugar control standards is less than GA20.0\% in guidelines, but from the calcification of vascular appearance of this time, less than GA18.0\% is considered necessary.

Keywords: Carotid artery calcified plaque, Carotid artery ultrasound test, GA (Glycoalbumin), Serum phosphorus level.

\section{0}

\section{Continuous Renal Replacement Therapy in Pediatric Pacients at the Hospital Infantil De Mexico Federico Gomez}

M. Delvillar-Vilchis Jr., L. Velasquez Jones, S. Valverde Rosas,
I. Del Moral

Pediatric Nephrology, Hospital Infantil De México Federico Gómez, Mexico City, Mexico

Background: Continuous renal replacement therapies (CRRT), is effective for the management of patients with hemodynamic instability and acute kidney injury. About $4 \%$ of these patients requires CRRT.

Methods: A retrospective, cross-sectional study. We obtain records of 16 patients were reviewed for the period from May 1, 2013 to July 31, 2014, in the pediatric intensive care unit (PICU).

Results: 16 patients, 8 men and 8 women ranging in age from 7 months to 18 years (mean 8 years) were analyzed. Registered weight were $7.6 \mathrm{~kg}$ to $60 \mathrm{~kg}$ (average $23.7 \mathrm{~kg}$ ) All with aminergic and vasopressor support, $13(81.25 \%)$ with more than two drugs, all mechanically ventilated. The etiologies were: by pass cardiac surgery in $8(50 \%)$, oncologic urgencies $4(25 \%)$, septic shock 3 (19\%), nephrotic syndrome $1(6 \%)$. The indications of the beginning of the administration were CRRT oligoanuria, fluid overload, and metabolic acidosis.

The CRRT used: venous-venous hemofiltration in $9(60 \%)$, venous-venous Hemodialfitration 2 (13\%), both in $4(27 \%)$. The treatment time 4 patients less than $10 \mathrm{hrs}$; suspended for hemodynamic instability, 5 patients for $12 \mathrm{hrs}, 3 \mathrm{hrs} 24$ patients, 3 patients and 1 patient 36 hrs $60 \mathrm{hrs}$. Used filters Helixone 12 (75\%), F60A 4 (25\%), baby lines (1), pediatric (11) and adult (4). All with fluid replacement and prefilter replacement rate: $20 \mathrm{ml} / \mathrm{min} 14(87.5 \%)$, $45 \mathrm{ml} / \mathrm{min}^{2}(6.25 \%)$, and $70 \mathrm{ml} / \mathrm{min}^{2}(6.25 \%)$. The average ultrafiltration (3475) $\mathrm{ml} \mathrm{SD}$. Clinical outcomes was death 10 (63\%), and improvement (37\%).

Conclusions: Administration of CRRT improves patient survival, coinciding with the average reported in the literature. The treatment time was inadequate, however because it is a surrogate service, it was not possible to adjust properly.

Keywords: Continuous renal replacement therapy, Pediatric pacients. 


\section{1 \\ Sodium Polystyrene Sulfonate Is More Effective in Lowering Potassium Than 1 K Bath in Chronic Hemodialysis Patient}

\section{Ziad Arabi, Jeffry Rimmer, Alan Segal}

Division of Nephrology at University of Vermont, Burlington, VT, USA

Hyperkalemia in is common in hemodialysis patients and it is associated with increased mortality [1]. Treatment can be challenging especially in patients who are not adherent to dietary potassium restriction. In these cases, treatment options include altering potassium bath from standard bath $(2 \mathrm{mEq} / \mathrm{L} \mathrm{K})$ to lower potassium bath $(1 \mathrm{mEq} / \mathrm{L} \mathrm{K})$ which will significantly remove more potassium $\{(62.9 \pm 5.1 \mathrm{mEq})$ for the $2-\mathrm{K}$ dialysate $(50.6 \pm 6 \mathrm{mEq})$, and the $1-\mathrm{K}$ dialysate $\}$ [2]. However rapid potassium shifts have been associated with cardiac arrhythmias [3].

Another option is to administer Sodium Polystyrene Sulfonate (Kayexalate); a cation-exchange resin which can be administered orally or in an enema. This medication has an in vitro exchange capacity of approximately $3.1 \mathrm{mEq}$ (in vivo approximately $1 \mathrm{mEq}$ ) of potassium per gram. The sodium content is approximately $100 \mathrm{mg}(4.1 \mathrm{mEq})$ per gram of the drug. Sodium ions which are released and are replaced by potassium ions mainly in the large intestine. The efficiency of this process is limited and variable. It is approximately 33 percent but the range is large. Effective lowering of serum potassium with Kayexalate may take hours to days [4]. Gastrointestinal side effects are common and serous GI side effects (including cases of intestinal necrosis) have been reported [5].

The potassium lowering effects of low potassium dialysate and potassium binding resin have not been compared in hemodialysis patients. Based on these estimations it is expected that the administration of Kaxyalate is more effective in lowering serum potas- sium than altering dialysis bath potassium concentration from $2 \mathrm{mEq} / \mathrm{L}$ to $1 \mathrm{mEq} / \mathrm{L}$. Here we report an observation that supports these findings.

This is a 54 year old man with history of ESRD treated with hemodialysis who was admitted in March 2014 for CABG. His course was complicated with cardiac arrest and prolonged hospitalization. Post-operative mental status changes impaired adherence to a dietary potassium restriction resulting in the use of a $1 \mathrm{mEq} / \mathrm{L}$ potassium bath from March until mid of May 2014 (see 1). In mid-May the patient seemed to more carefully follow a potassium restriction. His potassium improved and he was switched to $2 \mathrm{mEq} / \mathrm{L}$ bath (see 2). His serum potassium increased to 6.7 and patient was returned to a $1 \mathrm{mEq} / \mathrm{L} \mathrm{K}$ bath (see 3). The serum potassium concentration improved but remained elevated. Kayexylate, $15 \mathrm{G}$ by mouth daily was started (from 6/7/2014 till 6/20) (see 4). This resulted in a marked reduction of serum potassium levels. Two weeks of oral kayexalate appeared to deplete his total body potassium sufficiently that his potassium remained at acceptable levels for few days even after stopping kayexalate and using higher potassium bath ( $2 \mathrm{mEq} / \mathrm{L}$ from $6 / 20 / 14$ to $6 / 28 / 2014$ ) (see 5). On 6/28 the serum potassium rose to 6.9 and the patient was returned to a $1 \mathrm{~K}$ $\mathrm{mEq} / \mathrm{L}$ bath (see 6).

The patient remained non-adherent to $\mathrm{K}$ restriction and his potassium remained elevated with multiple readings more than 6 (see 6). Because of the concerns of the risk of arrhythmia from chronic use of lower $\mathrm{K}$ bath in a patient with recent $\mathrm{CABG}$ and cardiac arrest, he was changed to a $2 \mathrm{mEq}$ bath on 7/20/214 and kayexalate $30 \mathrm{mg}$ three times/week on non-dialysis days was administered (see 7). The serum potassium improved but patient declined to take any more kayexalate because of GI intolerance and a $1 \mathrm{mEq} / \mathrm{L}$ bath (see 8 ) was restarted.

The patient's mental status gradually improved and he was able to follow dietary restriction more carefully. Serum potassium remained in an acceptable range except the need for one dose of

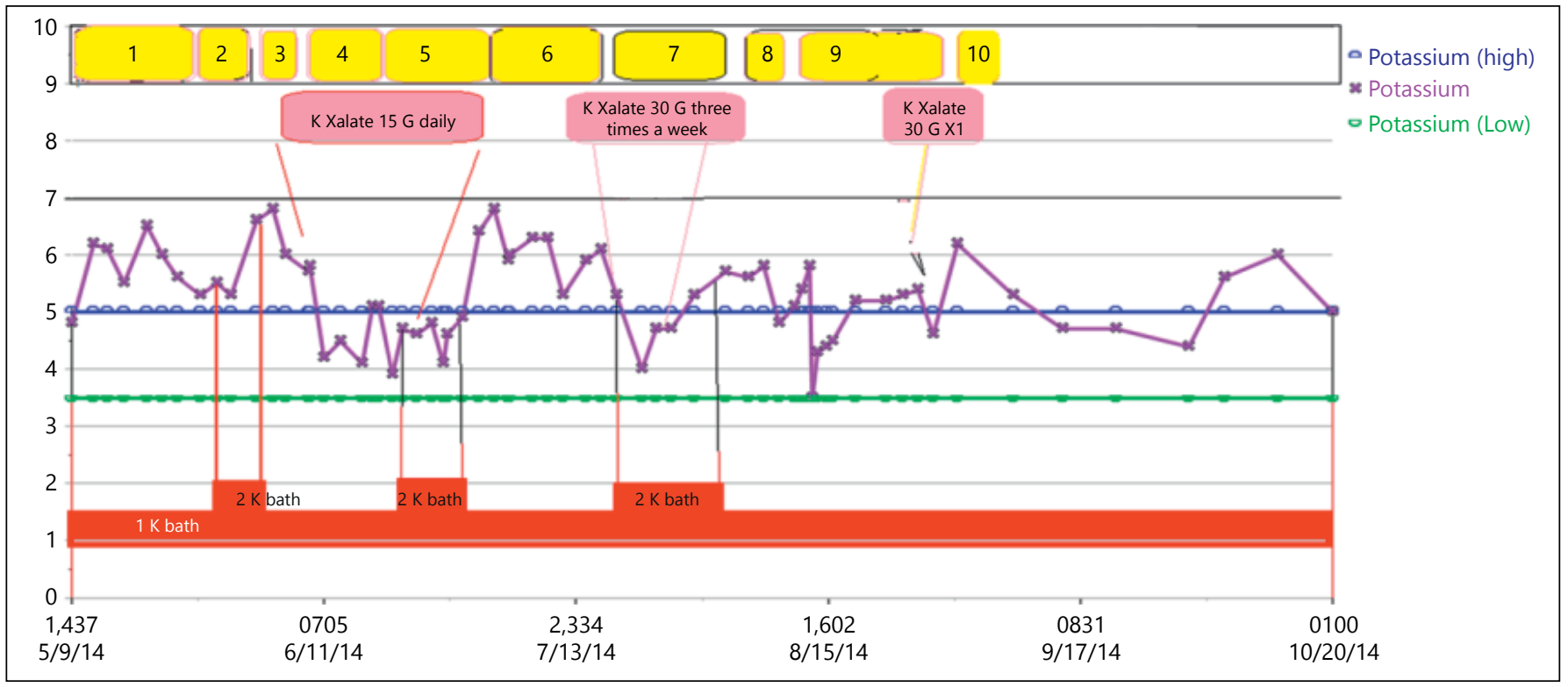

Fig. 1. (for Abstract 31). 
kayexalate (15 G) on $8 / 27 / 14$ (see 9). Patient was discharged to rehabilitation on $8 / 31 / 14$ (see 10$)$.

Conclusion: The administration of Sodium Polystyrene Sulfonate might be more effective in lowering the potassium than using lower potassium bath $(1 \mathrm{~K})$. Until randomized control study to confirm this observation, patients and nephrologists need to continue to weigh the potential side effects of each modality.

\section{References}

1 Kovesdy C, et al: Serum and Dialysate Potassium Concentrations and Survival in Hemodialysis Patients. Clin J Am Soc Nephrol 2007;2:999_ 1007

2 Hou S1, McElroy PA, Nootens J, Beach M: Safety and efficacy of lowpotassium dialysate. Am J Kidney Dis 1989;13:137-143.

3 Di Iorio B1, et al: Dialysate bath and QTc interval in patients on chronic maintenance hemodialysis: pilot study of single dialysis effects. J Nephrol 2012;25:653-660.

4 Kayexalate - FDA prescribing information, side effects http://www.drugs. com/pro/kayexalate.html\#i4i_warnings_id_cc171713-6d46-484d-ad83f0b7d8e0f8c9.

5 Rogers FB, Li SC: Acute colonic necrosis associated with sodium polystyrene sulfonate (Kayexalate) enemas in a critically ill patient: case report and review of the literature. J Trauma 2001;51:395-397.

\section{Dialysis: Anemia and Iron Metabolism}

32

\section{The Erythropoietin Saga-using Clinical Controlled Trials To Create A False Narrative}

\section{B. von Hartitzsch}

Nephrology Specialists of Oklahoma, Tulsa, OK, USA

Background: Academic Medicine, Statisticians and the FDA have used the NHCT Trial, the CHOIR Trial and the TREAT Trials to create a false narrative that correcting Hemoglobin levels to the normal range $13-16 \mathrm{gm} / \mathrm{dl}$ causes thrombosis, Myocardial infarcts, Strokes and Deaths. Clinical controlled trials come with instructions to ensure that the results and conclusions are valid -The Validity tests. Did randomization affect the results? Were there extraneous factors that influenced the results? After applying these tests to the three trials the question was how have so many supposedly intelligent people ignored important statistical data and made so many mistakes? The only logical answer is they had assumed that the hemoglobin's of the treated group had all gone up, events and deaths had to be caused by thrombosis secondary to the higher hemoglobin. That did not happen.

Methods: In each trial there was an excess of sicker patients with erythropoietin resistance who failed to respond with a rise in Hemoglobin and who in fact died from ischemia due to their low hemoglobin. In the Treat trial excesses of history of stroke and atrial fibrillation at randomization eliminated 36 of the 48 extra strokes allowing erythropoietin resistance and anemia to account for the rest. In addition the Nephrologists' lack of understanding of the physiology and function of hemoglobin and its oxygen carrying capacity has lead to repetition of the same false conclusion in each trial that it is unnecessary to raise the hemoglobin levels to normal because a smaller increase in the oxygen carrying capacity of the blood, also appreciated as an improved well being, resulted in similar answers for the quality of life questions. When both groups have the hemoglobin, the oxygen carrying capacity of their blood, raised a measurable test of exercise endurance on a treadmill is required to compare the effect of the two levels of treatment.

Results: The assumption that higher hemoglobin levels cause more events and deaths is fiction not supportable by any factual data. The actual facts say the opposite - Hemoglobin's $>13.3$ have 6 times fewer events than hemoglobin's below 10.9 gm/dl FDA data.

Conclusions: When we start treating anemia when the hemoglobin first drops out of the normal range when erythropoietin resistance, erythropoietin doses and cost would be at minimum, patients will be healthier, able to remain employed, more productive and we will prevent the need for dialysis in most patients in 10 years. In contrast the false narrative to justify rationing and to reduce Medicare and Health care costs has resulted in epidemics of cardiac disease, renal disease, and dialysis patients.

Keywords: Preventing dialysis, FDA-false narrative, Erythropoietin-truth, No fiction.

\section{Glomerular and Tubulointerstitial Disorders}

33

Use of C4d Biomarker as a Diagnostic Tool to Classify
Membranoproliferative Glomerulonephritis

Nirupama Gupta ${ }^{1}$, Dara Wakefield ${ }^{2}$, William Clapp ${ }^{2}$, Eduardo H. Garin ${ }^{1}$

'Division of Nephrology, Department of Pediatrics, ${ }^{2}$ Division of Pathology, Department of Pathology, Immunology and Laboratory Medicine, College of Medicine, University of Florida, Gainesville, FL, USA

Background: Membranoproliferative glomerulonephritis (MPGN) in 2013 was reclassified as MPGN and C3 glomerulopathy $(\mathrm{C} 3 \mathrm{G})$ based on its pathogenesis involving the complement cascade, either the classical or alternative pathway respectively. This study aimed to evaluate whether C4d biomarker, a cleavage protein of $\mathrm{C} 4$, could be used as a diagnostic tool to try to differentiate between classical or alternative pathway activation in MPGN, and possibly guide in more appropriate molecular testing.

Methods: We conducted a retrospective study including adult and pediatric patients diagnosed with MPGN and minimal change disease (MCD) from January 2000 to December 2012 found by using the Informatics for Integrating Biology and the Bedside software. Pertinent data from chart review were recorded. The formalin-fixed paraffin-embedded tissues were stained for C4d deposition using rabbit anti-human C4d polyclonal antibody via an immunoperoxidase method. Statistics were done using MannWhitney and chi-squared tests. 
Results: Sixteen MPGN and 9 MCD biopsies were included in the study. The control MCD biopsies had none or minimal C4d staining. Using the 2013 C3G consensus classification, the MPGN group was sub-classified as: MPGN (8), C3G (7), and unclassified MPGN (1)

Based on C4d immunohistochemical staining, 13 biopsies had C4d glomerular staining and 3 patients did not. Seven of these biopsies had $\mathrm{Clq}(+)$ and $\mathrm{C} 4 \mathrm{~d}(+)$ (classical pathway activation) and 6 had C4d (+) and C1q (-) (lectin pathway activation). The lectin pathway was presumed to be involved in 3 of the MPGN and 3 of the $\mathrm{C} 3 \mathrm{G}$ biopsies. The three $\mathrm{C} 4 \mathrm{~d}$ negative biopsies had evidence of alternative pathway activation.

Of the 8 MPGN biopsies reclassified by the 2013 C3G consensus classification, 2 were reclassified as $\mathrm{C} 3 \mathrm{G}$; of the six C3G biopsies, 3 were reclassified as MPGN; and the unknown biopsy was reclassified as MPGN after C4d staining.

Conclusions: C4d stain can help differentiate between MPGN and $\mathrm{C} 3 \mathrm{G}$ and may possibly guide in additional molecular testing for certain patients. This study also reports for the first time that the lectin pathway also seems to play a prominent role in the pathogenesis of MPGN in some patients.

Keywords: C4d, MPGN.

\section{4}

Glomerular Disorders in Two Patients with Intestinal Transplant

\author{
A. Nada ${ }^{1}$, P. Backer ${ }^{2}$, B. Becknell ${ }^{1}$, H. Patel ${ }^{1}$ \\ ${ }^{1}$ Pediatric Nephrology, Nationwide Children's Hospital, \\ Columbus, $\mathrm{OH}, \mathrm{USA}{ }^{2}$ Division of Pathology, Nationwide \\ Children's Hospital, Columbus, $\mathrm{OH}$, USA
}

Background: Prior studies of end stage kidney disease (ESKD) after intestinal transplant (IT) found the incidence of ESKD is up to $3 \%$ of children and $21 \%$ of adults. ESKD is often attributed to calcineurin inhibitors in children and diabetes and hypertension in adults, but reports of glomerular diseases in this population is rare. To our knowledge this is the first report of the pathological findings of glomerular disease in patients who received IT as children.

Methods: We report 2 patients with IT for Hirschsprung disease who had kidney biopsy for proteinuria. The age at first IT was 2 and 16 years. Neither patient had diabetes mellitus at the time of the kidney biopsy. Both patients had progressive increase in proteinuria with urine protein/creatinine ratio of $15 \mathrm{mg} / \mathrm{mg}$ in the first patient and $30 \mathrm{mg} / \mathrm{mg}$ in the second patient at the time of biopsy. The estimated glomerular filtration rate at the time of the biopsy was 55 and $33 \mathrm{ml} / \mathrm{min} / 1.73 \mathrm{~m}^{2}$. Both kidney biopsies showed focal segmental glomerulosclerosis (FSGS) with hyalinosis on light microscopy. Both had positive IgG, IgM, IgA, C3, C1q, $\mathrm{C} 4$, and fibrinogen on immunofluorescence. Electron microscopy showed primarily subendothelial deposits in the first patient and a small amount of subepithelial and intramembranous deposits in the second. Both patients had normal serum C3, and C4 complement.

Results: NA.

Conclusions: We found FSGS with immune deposits in 2 patients with IT who developed proteinuria. This report underscores the importance of obtaining kidney biopsy in patients with
IT who develop proteinuria, especially when the proteinuria is out of proportion to what one may expect from drug toxicity. Early screening and identification of underlying kidney disease may allow intervention to prevent or slow the development of ESKD in these children. Studying larger number of patients to further investigate our preliminary findings and develop a better understanding of the cause of ESKD in these patients is warranted.

Keywords: FSGS, Intestinal, Transplant.

\section{5 \\ Effectiveness of Prednisone-Tacrolimus Combination Compared with Prednisone-Cyclosporine for Treating Steroid-Resistant Nephrotic Syndrome in Pediatric Patients}

\author{
R.G. Guerrero-Kanan Jr., I.E. Del Moral Espinosa, \\ L. Velasquez Jones, S. Valverde Rosas, M. Medeiros, \\ G. Ramon Garcia
}

Pediatric Nephrology, Hospital Infantil de Mexico Federico Gomez, Mexico City, Mexico

Background: About 5\% of children with idiopathic nephrotic syndrome (NS) present steroid-resistant nephrotic syndrome (SRNS) with the presence of focal segmental glomerulosclerosis (FSGS). We need more studies that demonstrate that treatment with Prednisone (PDN) and Tacrolimus (FK) in pediatric patients with SRNS for a period of 24 months have a higher frequency of complete or partial remissions in relation to the standard treatment with prednisone and Cyclosporine $(\mathrm{CyA})$. We also aimed to compare the histologic characteristics after a year of treatment.

Methods: A retrospective, descriptive and comparative clinical study was performed in children with SRNS.

Prednisone $60 \mathrm{mg} / \mathrm{m}^{2} /$ day, during the 1 month followed by $30 \mathrm{mg} / \mathrm{m}^{2} /$ day every $48 \mathrm{~h}$ for 5 months. Group I received CyA $5 \mathrm{mg} / \mathrm{kg} /$ day. Group II receive FK $0.1-0.2 \mathrm{mg} / \mathrm{kg} /$ day.

Results: We included 16 pediatric patients with similar characteristics ( 9 received treatment with CyA and 7 received treatment with FK). The initial histologic characteristics showed focal segmental glomerulosclerosis (FSGS) in 57\%, diffuse mesangial proliferation (PMD) in $28 \%$ and minimal changes (MCG) in $33 \%$. In the short-term, FK appeared to be better than CyA by inducing complete remission at three months in $57 \%$ and $44 \%$ of patients respectively. However, the difference in outcomes between the Cya and FK therapy was not stadistical different in the two years (X2: 0.423 p: 0.809 and X2: 3.810 , p: 0.149$)$ After 12 months of treatment, the percentage of complete remission was $85 \%$ in the FK group and $77 \%$ in the CyA group, no stadistically significant (X2 $0.163 \mathrm{a} P, \mathrm{p}=0.687)$. A greater proportion of patients receiving CyA relapsed compared with tacrolimus (P: 0.04). The mean duration of therapy at the time of relapse was 31 weeks in the FK group and 18 weeks in the group of CyA. We could not demonstrate any valid association. It was observed that patients in the tacrolimus group had a lower incidence of nephrotoxicity, dyslipidemia, and hypertension.

Conclusions: Although patients receiving FK showed lower relapse rates, and significantly lower in the first year of treatment, the relapse rate in subsequent years were the same, showing no 
more effectiveness in inducing remission at 2 years. FK is a drug that has the advantage of being less nephrotoxic, fewer adverse reactions, and lower degree of dyslipidemia. There were no differences in the histological characteristics reported in before and after the first year of treatment in both groups.

Keywords: Steroid resistant, Nephrotic syndrome, Tacrolimus, Cyclosporine.

\section{Hypertension}

\section{6 \\ Neonatal Hypertension: Improving Blood Pressure Measurement and Documentation in the Nicu}

H.A. Munoz, J. Samuel, C. Bell, A. Khan

University of Texas Houston, Houston, TX, USA

Background: While the gold standard for blood pressure (BP) is intra-arterial measurement, normative thresholds for BP in neonates is based on oscillometric devices. Previous studies have shown an inconsistent relationship between invasive and noninvasive BP. It is important to maintain a standardized protocol to minimize these inconsistencies and maximize accuracy in $\mathrm{BP}$ measurement.

Methods:

Primary Objective: To compare the documentation rate of BP measurements in the NICU before and after an educational intervention.

Secondary objectives: To determine whether any factors affect the difference between invasive and non-invasive BP such as inappropriate documentation, type of device, gestational age, infant weight, presence of hypotension and age of the intra-arterial line.

Design: We collected baseline data from 40 patients with intraarterial lines admitted to a level III NICU. An educational intervention was given to the NICU staff involved in obtaining and documenting BP measurements. Proper documentation was defined as simultaneous invasive and non-invasive BP recorded with patient's level of activity, upper extremity BP measurement, cuff size and invasive line location. Data collection post-intervention to determine the efficacy of the educational session is ongoing.

Results: We collected 1,322 paired invasive and non-invasive BP measurements during the pre-intervention period. 167 (12\%) oscillometric BP measurements and $143(10.8 \%)$ invasive measurements were properly documented. 17 (1.28\%) of the BP measurements had simultaneous complete documentation for both invasive and non-invasive BP.

Conclusions: There is a lack of proper documentation of BP measurements in the NICU but whether appropriate documentation affects BP measurement accuracy is yet to be determined.

Keywords: Blood pressure, Neonatal, QI.
37

\section{Interleukin-6 Inhibition Attenuates Renal Damage Without Altering Hypertension In Dahl Salt Sensitive Rats}

\author{
S. Hashmat ${ }^{1}$, N. Rudemiller ${ }^{2}$, H. Lund ${ }^{2}$, S. Van Why ${ }^{1}$, D.L. Mattson ${ }^{2}$ \\ ${ }^{1}$ Pediatric Nephrology, Medical College of Wisconsin, \\ Wuawatosa, WI, USA; ${ }^{2}$ Physiology, Medical College of \\ Wisconsin, Milwaukee, WI, USA
}

Background: Infiltrating immune cells in the kidney amplify hypertension and kidney damage in Dahl Salt Sensitive rats (SS/ Mcw). Dahl SS rats exhibit many phenotypic characters in common with human hypertension. Compared to circulating T-lymphocytes, infiltrating T cells in the kidney of Dahl SS rats exhibit increased mRNA for a variety of inflammatory cytokines; especially, infiltrating $\mathrm{T}$ cells have a 54 fold increase in interleukin (IL-6) mRNA. This study was performed to determine the role of IL-6 in the development of salt-sensitive hypertension and renal damage.

Methods: Experiments were performed on male Dahl SS rats ( $n=6-12 /$ group). Starting at 9 weeks of age, the rats were switched from a low $(0.4 \% \mathrm{NaCl})$ to a high salt diet $(4.0 \% \mathrm{NaCl})$ and administered goat anti-rat IL-6 neutralizing antibody (anti-rIL-6; dose $4 \mu \mathrm{g} /$ day, IP) (R\&D Systems, Minneapolis) or normal goat IgG control (dose $4 \mu \mathrm{g} /$ day, IP) for 3 weeks. The rats were instrumented with chronic indwelling femoral artery catheters and parameters of hypertension and renal disease were obtained in the conscious state after 3 weeks on the $4.0 \% \mathrm{NaCl}$ diet.

Results: The average mean arterial blood pressure (MAP) in Dahl SS rats administered anti-rIL-6 was not significantly different compared with control group $(136 \pm 2 \mathrm{~mm} \mathrm{Hg}$ vs $139 \pm$ $3 \mathrm{~mm} \mathrm{Hg}$, respectively) after three weeks of high salt. In contrast, the albumin excretion rate, an index of renal damage, was significantly attenuated in the anti-rIL- 6 group $(25 \pm 1$ and $52 \pm 4 \mathrm{mg} /$ day) than in the control group ( $42 \pm 6$ and $76 \pm 12 \mathrm{mg} /$ day) after 1 and 3 week period of high salt intake, respectively. Urine sodium, potassium and creatinine excretion rates were not different among the groups. The IL- 6 level in the homogenized renal cortex tended to be lower in the anti-rIL-6 group (1849 \pm 188 vs. $2010 \pm 135$ pg/ $\mathrm{ml})$ but did not reach significance.

Conclusions: The present study indicates that IL-6 mediates renal disease associated with salt sensitive hypertension independent of changes in arterial blood pressure.

Keywords: Interleukin-6, Hypertension, Dahl salt sensitive rats. 


\section{Bioengineering and Informatics}

38

\section{Assessing the Change in Provider Behavior Following the Introduction of a New Hypertension Recognition Tool}

M. Semanik

Nephrology, Seattle Children's Hospital, Seattle, WA, USA

Background: Pediatric hypertension is a significant and growing problem. However, it is often under-recognized in the outpatient, inpatient, and emergency settings. Several hypertension recognition tools have been developed, with varying levels of success in promoting the identification of elevated blood pressures. These tools were office-based and did not incorporate electronic medical records, which are increasingly used to provide care in ambulatory and inpatient settings. The purpose of this study is to assess the utility of incorporating a hypertension recognition tool into the electronic medical record.

Methods: A retrospective chart review will be performed, identifying patients aged 1-18 years seen at outpatient subspecialty clinics in a tertiary-care hospital who have elevated blood pressures (prehypertension or hypertension, as defined by the Fourth Report). The patients will be divided into two groups: 1) All patients with elevated blood pressures for a 3 month time period prior to the initiation of a hypertension recognition tool, and 2) All patients with elevated blood pressure for a 3 month time period following the initiation of the tool. There will be a 2 month 'washout' period in order to account for providers becoming accustomed to the new tool. The tool itself consists of reporting blood pressure percentiles (both systolic and diastolic) to providers; these will be displayed with other vital signs in the electronic medical record. The two groups will then be assessed for differences in outcomes tied to hypertension recognition: diagnosis of elevated blood pressure or hypertension; frequency of rechecking blood pressures; frequency of nephrology referrals; frequency of antihypertensive medication initiation; frequency of obtaining renal ultrasounds; and frequency of obtaining echocardiograms. Exclusion criteria consist of patients with known renal disease, patients with known cardiac disease, and patients with a prior diagnosis of hypertension.

Results: Data is being acquired as I write this, and will hopefully be available in several months!

Conclusions: Data is being acquired as I write this, and will hopefully be available in several months!

Keywords: Hypertension recognition, Informatics, Pediatric hypertension.

\section{Pharmacokinetics, Pharmacodynamics, and Pharmacogenetics}

\author{
39 \\ What Is Adequate Amount or Treatment Time of \\ Hemodialysis in a Case Suspicious for Baclofen \\ Intoxication: Case Report and Literature Review in \\ Pharmacokinetic Aspects \\ Y. Kim \\ Division of Nephrology, Department of Internal Medicine, \\ University of New Mexico, Albuquerque, NM, USA
}

Introduction: Baclofen is FDA approved to treat spasticity associated with various conditions including spinal cord lesion. With low biotransformation in the body, its route of excretion is mostly renal in unchanged form, posing patients with impaired kidney function at risk of overdose. Unlike alcohol-related intoxications in which on site monitoring and determination of elimination rate constant is feasible, baclofen plasma concentration is not readily available in timely fashion even at tertiary hospital and it is difficult to predict plasma concentration due to its variable volume of distribution, little known drug clearance in renal failure, and unclear medication history in many cases. As studying the case in pharmacokinetic aspects, A question was raised that what is adequate amount or treatment time of hemodialysis therapy in a case suspicious for baclofen intoxication but with drug plasma concentration which will be pending for a week, arbitrary 4 hours of hemodialysis would be enough?

Case Report and Analysis: A case of encephalopathy suspicious for baclofen toxicity was encountered and the patient was treated successfully with 4 hours of hemodialysis. Total elimination rate constant during hemodialysis was obtained with pre and post hemodialysis plasma concentrations using first order rate law equation. Initial plasma concentration was predicted based on medication administration record and observed clearance of baclofen in renal failure from previously published pharmacokinetic data.

Discussion: Literature review resulted in many case reports including case series. Undoubtedly, hemodialysis enhanced drug elimination in the cases with renal failure as well as normal renal function. Initial baclofen plasma concentrations in case reports ranged $0.25 \mu \mathrm{g} / \mathrm{ml}$ to $1.2 \mu \mathrm{g} / \mathrm{ml}$ with or without renal failure. A case of $17 \mu \mathrm{g} / \mathrm{ml}$ plasma concentration resulted in fatality. Plasma concentration in our case was predicted $0.81 \mu \mathrm{g} / \mathrm{ml}$ to $1.5 \mu \mathrm{g} / \mathrm{ml}$ using following equation, maintenance dose $=\mathrm{CL} \times \mathrm{C}_{\mathrm{p}} / \mathrm{F} .0 .89 \mu \mathrm{g} / \mathrm{ml}$ was actual value. Hemodialysis elimination rate constant ranged 0.15 $\mathrm{h}^{-1}$ to $2.9 \mathrm{~h}^{-1}$ with similar hemodialysis prescriptions. $0.21 \mathrm{~h}^{-1}$ was calculated in our case. To lower concentration of baclofen to mid therapeutic range, 3.4-10.7 h of hemodialysis therapy seems to be required with conservative clearance in frequent clinical scenarios. Plotting of drug concentration and elimination rate constant is prepared.

Keywords: Baclofen intoxication, Hemodialysis, Pharmacokinetics. 
40

Recurrence of Focal Segmental Glomerular Sclerosis (FSGS) in Kidney Allograft Pediatrics Recipients in the Hospital Infantil de México Federico Gómez

V.M. Barajas Valencia, L. Velasquez Jones, S. Valverde Rosas, J.C. Romo Vazquez

Pediatric Nephrology, Hospital Infantil de México Federico Gómez, Distrito Federal, Mexico

Background: Focal Segmental Glomerulosclerosis (FSGS) is the second cause of idiopathic nephrotic syndrome in children, progresses to end-stage renal disease (ESRD). Recurrence after kidney transplantation is presented in $30-50 \%$ and graft loss in $40-60 \%$. There are different treatments for recurrence and before transplantation, which is still controversial.

Methods: We report three cases of pediatric patients post-renal transplantation, with recurrent FSGS, determined as the presence of proteinuria by spot urine protein/creatinine ratio $>2.0 \mathrm{mg} / \mathrm{mg}$, independently of renal function, with biopsy that showed recurrence of disease. Serial measurements of spot urine protein/creatinine ratio were performed as follow-up. Plasmapheresis was performing between 5 to 20 sessions and a dose of rituximab $375 \mathrm{mg} / \mathrm{m}^{2} \mathrm{SC}$ at the end of plasmapheresis therapy. The patients were followed-up as outpatient. Immunosuppression with tacrolimus was used in two of them and the other one with cyclosporine.

Results: Two of the three patients, complete remission of proteinuria was achieved with the use of plasmapheresis associated with rituximab and the use of calcineurin as maintenance therapy. One patient had partial remission with proteinuria $(1.1 \mathrm{mg} / \mathrm{mg})$. All three patients appeared to have stable graft function by follow-up at 3 months.

Conclusions: Recurrence of FSGS in renal transplant patients has been increasing. And there is still controversy in the management of these patients in the acute phase of the disease and the type of immunosuppressive regimen. In our case report we observed that all had either complete or partial remission with this treatment scheme, unlike that reported in the literature with a margin of $64-70 \%$ of effectiveness. It is necessary to make futher studys like meta-analysis to determine treatment response.

Keywords: Kidney allograft, Focal segmental glomerulosclerosis, Recurrence disease.
41

\section{ANCA Vasculitis Presenting as Microangiopathic Anemia and Renal Insufficiency}

\section{A. Patil}

Pediatric Nephrology, Medical College of Wisconsin, Wauwatosa, WI, USA

Background: A 12 year old girl presented with history a month prior of non-specific abdominal pain, when several other family members had a prominent diarrheal illness. She subsequently developed progressive fatigue and pallor. Initial labs showed severe anemia with schistocytes on smear, but normal platelet count. Blood creatinine $(\mathrm{Cr})$ was $3.87 \mathrm{mg} / \mathrm{dl}$. Chest X-ray was normal.

Methods: Initial presumptive diagnosis was late presentation of hemolytic uremic syndrome (HUS), in resolution phase. After initial improvement in $\mathrm{Cr}$ and stable $\mathrm{CBC}$ over a week, she developed worsening $\mathrm{Cr}$, progressive dyspnea, worsening anemia and new infiltrates indicative of pulmonary hemorrhage. Renal biopsy showed diffuse crescentic glomerulonephritis. She was treated with pulse steroid, oral Cytoxan and plasma exchange. She later developed persistent thrombocytopenia with return of schistocytes on peripheral smear. Microangiopathic features then spontaneously resolved in a week. Her pulmonary hemorrhage resolved, but she progressed to ESRD. Anti-myeloperoxidase levels returned high. Genetic testing for mutations associated with atypical HUS was negative.

Conclusions: This case illustrates an unusual presentation of microscopic polyangiitis, without evident pulmonary involvement at presentation, that suggested HUS as initial diagnosis. Microangiopathic anemia, then, may be a presenting and prominent feature of microscopic polyangiitis.

Keywords: Microscopic polyangiitis, Microangiopathic anemia, Vasculitis. 


\section{Vascular Biology}

\section{2}

\section{Cardiovascular Manifestations of Aberrant Elastic Fiber Formation in Mice with Mutant Fibulin-4}

\author{
C.M. Halabi ${ }^{1}$, M. Chu ${ }^{2}$, R.P. Mecham ${ }^{3}$
}

${ }^{1}$ Pediatrics, Washington University School of Medicine, Saint Louis, MO, USA; ${ }^{2}$ Dermatology and Cutaneous Biology, Thomas Jefferson University, Philadelphia, PA, USA ; ${ }^{3}$ Cell Biology and Physiology, Washington University School of Medicine, Saint Louis, MO, USA

Background: Fibulin-4 (FBLN4) is an extracellular matrix protein required for proper elastic fiber formation. To date, several individuals carrying homozygous or compound heterozygous mutations in FBLN4 have been described. While most individuals present at birth with abnormal facies and inelastic/redundant skin, the variable systemic manifestations are most striking and devastating. These include arterial tortuosity, ascending aortic aneurysms, emphysema, diaphragmatic and inguinal hernias, among others. To better understand the effect of mutant Fbln 4 on elastic fiber formation and resulting pathologic manifestations, a knock-in mouse model carrying a disease-causing mutation in Fbln4 (E57K) was generated.
Methods: Cardiovascular anatomy of 6 week-old to 7 monthold Fbln4E57K mice and littermate controls (WT) was assessed by visual inspection, histology and electron microscopy (EM) of large and small arteries, and echocardiography. In addition to echocardiography, functional consequences of Fbln4E57K were determined by measuring arterial blood pressure and large artery compliance in 3 to 7 month-old mice.

Results: Arterial tortuosity and elongation were noted in all Fbln4E57K mice, while aortic root dilatation and/or ascending aortic aneurysms were seen in nearly $50 \%$ of Fbln4E57K mice. Whereas aneurysms were limited to the ascending aorta, vascular elongation was noted along the arterial tree. Electron microscopy of large arteries showed patchy areas of significant elastic fiber fragmentation and disarray. On echocardiography, aortic insufficiency was noted in mice with aortic root dilatation, and while left ventricular systolic function was normal at 6 weeks, dilated cardiomyopathy developed in some animals by 6 months of age. All Fbln4E57K mice had systolic hypertension with pulse pressure widening as well as decreased large artery compliance.

Conclusions: In summary, E57K mutation in Fbln4 disrupts normal elastic fiber formation leading to significant detrimental cardiovascular consequences. Further characterization of this animal model will not only advance our understanding of the role of extracellular matrix in the development/pathophysiology of disease processes such as hypertension and thoracic aortic aneurysms, but will also shed light on the role of FBLN4 in elastic fiber formation.

Keywords: Elastic fiber, Hypertension, Vascular stiffness. 